\title{
Mechanisms of Acid-Promoted $\mathrm{N}_{2}$ and $\mathrm{N}_{2} \mathrm{O}$ Generation from $\mathrm{NH}_{2} \mathrm{NO}$ and $\mathrm{NH}_{2} \mathrm{NO}_{2}$
}

\author{
Tongheng Chen, Zhengyi Wan, Tarek Trabelsi, Chongqin Zhu* and Joseph \\ S. Francisco*
}

${ }^{1}$ Department of Earth and Environmental Sciences and Department of Chemistry, University of Pennsylvania, Philadelphia, Pennsylvania 19104, United States

\section{Supporting Information}

Table S1 lists the original calculated energies of all the reactants, transition states and intermediates of $\mathrm{NH}_{2} \mathrm{NO} / \mathrm{NH}_{2} \mathrm{NO}_{2}$ with water, formic acid or nitric acid in Hartrees. Table $\mathrm{S} 2$ shows the vibrational frequencies $\left(\mathrm{cm}^{-1}\right)$ and zero-point energy corrections $(\mathrm{kcal} / \mathrm{mol})$ from M06-2X/aug-cc-pVTZ of all the reactants, transition states, intermediates and products involved in the $\mathrm{NH}_{2} \mathrm{NO} / \mathrm{NH}_{2} \mathrm{NO}_{2}$ reaction system. Table $\mathrm{S} 3$ shows the relative energy of all the reactants, transition states, intermediates and products. Table S4 lists zero-point vibrational energy (ZPE) corrected binding energies of all related complexes at M06-2X and CCSD(T) levels of Theory. Table S5 lists all related species and complexes with optimized structures by Cartesian coordinates in M06-2X/aug-cc-pVTZ level. 
Table S1. Calculated Energies (Hartrees) of All the Reactants, Transition States, Intermediates and Products of $\mathrm{H}_{2} \mathrm{NNO} / \mathrm{H}_{2} \mathrm{NNO}_{2}$ with Catalysis of Water, Formic Acid and Nitric Acid at M06-2X Level including ZPE and CCSD(T) Level without ZPE. The Basis Sets are both aug-cc-pVTZ

\begin{tabular}{|c|c|c|}
\hline \multirow{2}{*}{ Species/Complexes } & M06-2X & $\operatorname{CCSD}(\mathrm{T})$ \\
\hline & aug-cc-pVTZ & aug-cc-pVTZ \\
\hline \multicolumn{3}{|l|}{ Single molecules } \\
\hline $\mathrm{N}_{2}$ & -109.528947 & -109.380023 \\
\hline $\mathrm{N}_{2} \mathrm{O}$ & -184.655026 & -184.419211 \\
\hline $\mathrm{H}_{2} \mathrm{O}$ & -76.408556 & -76.3422896 \\
\hline $\mathrm{HNO}_{3}$ & -280.869978 & -280.544040 \\
\hline cis- $\mathrm{HCOOH}$ & -189.728612 & -189.510709 \\
\hline trans- $\mathrm{HCOOH}$ & -189.735284 & -189.517432 \\
\hline $\mathrm{NH}_{3}$ & -56.518491 & -56.4805249 \\
\hline HONO & -205.688243 & -205.450469 \\
\hline \multicolumn{3}{|c|}{$\mathrm{NH}_{2} \mathrm{NO} \longrightarrow \mathrm{N}_{2}+\mathrm{H}_{2} \mathrm{O}$ (Figure 2) } \\
\hline $\mathrm{NH}_{2} \mathrm{NO}, 1$ & -185.816852 & -185.602783 \\
\hline $\mathrm{TS} 1,2$ & -185.764778 & -185.549551 \\
\hline $\mathrm{HNNOH}, 3$ & -185.816589 & -185.604513 \\
\hline $\mathrm{TS} 2,4$ & -185.802332 & -185.589134 \\
\hline $\mathrm{HNNOH}, 5$ & -185.814974 & -185.603421 \\
\hline $\mathrm{TS} 3,6$ & -185.759247 & -185.542634 \\
\hline $\mathrm{HNNOH}, 7$ & -185.814717 & -185.603477 \\
\hline $\mathrm{TS} 4,8$ & -185.773915 & -185.562336 \\
\hline $\mathrm{N}_{2}+\mathrm{H}_{2} \mathrm{O}$ & -185.938129 & -185.724728 \\
\hline \multicolumn{3}{|c|}{$\mathrm{NH}_{2} \mathrm{NO}+\mathrm{H}_{2} \mathrm{O} \longrightarrow \mathrm{N}_{2}+2 \mathrm{H}_{2} \mathrm{O}$ (Figure 4) } \\
\hline $\mathrm{NH}_{2} \mathrm{NO} \cdots \mathrm{H}_{2} \mathrm{O}, 1$ & -262.235647 & -261.958731 \\
\hline $\mathrm{TS} 1 \cdots \mathrm{H}_{2} \mathrm{O}, 2$ & -262.213575 & -261.929711 \\
\hline $\mathrm{HNNOH} \cdots \mathrm{H}_{2} \mathrm{O}, 3$ & -262.234513 & -261.959162 \\
\hline $\mathrm{HNNOH} \cdots \mathrm{H}_{2} \mathrm{O}, 16$ & -262.228690 & -261.953470 \\
\hline $\mathrm{TS} 3 \cdots \mathrm{H}_{2} \mathrm{O}, 17$ & -262.147775 & -261.863393 \\
\hline $\mathrm{HNNOH} \cdots \mathrm{H}_{2} \mathrm{O}, 18$ & -262.227513 & -261.952909 \\
\hline $\mathrm{HNNOH} \cdots \mathrm{H}_{2} \mathrm{O}, 25$ & -262.229358 & -261.954712 \\
\hline $\mathrm{TS} 4 \cdots \mathrm{H}_{2} \mathrm{O}, 26$ & -262.219289 & -261.942545 \\
\hline $\mathrm{N}_{2}+\mathrm{H}_{2} \mathrm{O} \cdots \mathrm{H}_{2} \mathrm{O}$ & -262.352560 & -262.076535 \\
\hline \multicolumn{3}{|c|}{$\mathrm{NH}_{2} \mathrm{NO}+\mathrm{HNO}_{3} \longrightarrow \mathrm{N}_{2}+\mathrm{H}_{2} \mathrm{O}+\mathrm{HNO}_{3}$ (Figure 4) } \\
\hline $\mathrm{NH}_{2} \mathrm{NO} \cdots \mathrm{HNO}_{3}, 4$ & -466.704716 & -466.166982 \\
\hline $\mathrm{TS} 1 \cdots \mathrm{HNO}_{3}, 5$ & -466.696568 & -466.151828 \\
\hline $\mathrm{HNNOH} \cdots \mathrm{HNO}_{3}, 6$ & -466.702654 & -466.166314 \\
\hline $\mathrm{HNNOH} \cdots \mathrm{HNO}_{3}, 10$ & -466.697751 & -466.160979 \\
\hline $\mathrm{TS} 2 \cdots \mathrm{HNO}_{3}, 11$ & -466.676675 & -466.131731 \\
\hline $\mathrm{HNNOH} \cdots \mathrm{HNO}_{3}, 12$ & -466.697556 & -466.161078 \\
\hline $\mathrm{HNNOH} \cdots \mathrm{HNO}_{3}, 19$ & -466.699435 & -466.163237 \\
\hline $\mathrm{TS} 3 \cdots \mathrm{HNO}_{3}, 20$ & -466.670462 & -466.127967 \\
\hline $\mathrm{HNNOH} \cdots \mathrm{HNO}_{3}, 21$ & -466.698506 & -466.162445 \\
\hline $\mathrm{HNNOH} \cdots \mathrm{HNO}_{3}, 27$ & -466.695820 & -466.160755 \\
\hline $\mathrm{N}_{2}+\mathrm{H}_{2} \mathrm{O} \cdots \mathrm{HNO}_{3}$ & -466.824108 & -466.286635 \\
\hline \multicolumn{3}{|c|}{$\mathrm{NH}_{2} \mathrm{NO}+\mathrm{HCOOH} \longrightarrow \mathrm{N}_{2}+\mathrm{H}_{2} \mathrm{O}+\mathrm{HCOOH}$ (Figure 4) } \\
\hline $\mathrm{NH}_{2} \mathrm{NO} \cdots \mathrm{HCOOH}, 7$ & -375.570884 & -375.142566 \\
\hline TS1 $\cdots \mathrm{HCOOH}, 8$ & -375.563276 & -375.125604 \\
\hline $\mathrm{HNNOH} \cdots \mathrm{HCOOH}, 9$ & -375.569210 & -375.141926 \\
\hline
\end{tabular}




\begin{tabular}{|c|c|c|}
\hline & & \\
\hline HNNOH $\cdots \mathrm{HCOOH}, 13$ & -375.563075 & -375.134920 \\
\hline $\mathrm{TS} 2 \cdots \mathrm{HCOOH}, 14$ & -375.543829 & -375.107608 \\
\hline $\mathrm{HNNOH} \cdots \mathrm{HCOOH}, 15$ & -375.563483 & -375.135850 \\
\hline HNNOH $\cdots \mathrm{HCOOH}, 22$ & -375.562466 & -375.135137 \\
\hline TS3 $\cdots \mathrm{HCOOH}, 23$ & -375.532768 & -375.096872 \\
\hline $\mathrm{HNNOH} \cdots \mathrm{HCOOH}, 24$ & -375.561182 & -375.134194 \\
\hline $\mathrm{HNNOH} \cdots \mathrm{HCOOH}, 28$ & -375.561505 & -375.135398 \\
\hline $\mathrm{N}_{2}+\mathrm{H}_{2} \mathrm{O} \cdots \mathrm{HCOOH}$ & -375.688292 & -375.259078 \\
\hline \multicolumn{3}{|c|}{$\mathrm{NH}_{2} \mathrm{NO}_{2} \longrightarrow \mathrm{N}_{2} \mathrm{O}+\mathrm{H}_{2} \mathrm{O}$ (Figure 6) } \\
\hline $\mathrm{NH}_{2} \mathrm{NO}_{2}, 1$ & -261.003687 & -260.704121 \\
\hline $\mathrm{TS} 1,2$ & -260.943314 & -260.637711 \\
\hline HNNOOH, 3 & -260.988906 & -260.689093 \\
\hline $\mathrm{TS} 2,4$ & -260.979189 & -260.679107 \\
\hline HNNOOH, 5 & -260.989373 & -260.689572 \\
\hline TS3, 6 & -260.948698 & -260.644256 \\
\hline HNNOOH, 7 & -260.988556 & -260.688803 \\
\hline TS4, 8 & -260.933081 & -260.634031 \\
\hline $\mathrm{N}_{2} \mathrm{O}+\mathrm{H}_{2} \mathrm{O}$ & -261.066792 & -260.765959 \\
\hline \multicolumn{3}{|c|}{$\mathrm{NH}_{2} \mathrm{NO}_{2}+\mathrm{H}_{2} \mathrm{O} \longrightarrow \mathrm{N}_{2} \mathrm{O}+2 \mathrm{H}_{2} \mathrm{O}$ (Figure 8) } \\
\hline $\mathrm{NH}_{2} \mathrm{NO}_{2} \cdots \mathrm{H}_{2} \mathrm{O}, 1$ & -337.423796 & -337.059934 \\
\hline $\mathrm{TS} 1 \cdots \mathrm{H}_{2} \mathrm{O}, 2$ & -337.399295 & -337.026428 \\
\hline $\mathrm{HNNOOH} \cdots \mathrm{H}_{2} \mathrm{O}, 3$ & -337.411844 & -337.048316 \\
\hline $\mathrm{HNNOOH} \cdots \mathrm{H}_{2} \mathrm{O}, 16$ & -337.412404 & -337.048578 \\
\hline TS3 $\cdots \mathrm{H}_{2} \mathrm{O}, 17$ & -337.398992 & -337.027420 \\
\hline $\mathrm{HNNOOH} \cdots \mathrm{H}_{2} \mathrm{O}, 18$ & -337.411979 & -337.048114 \\
\hline $\mathrm{HNNOOH} \cdots \mathrm{H}_{2} \mathrm{O}, 25$ & -337.403863 & -337.040469 \\
\hline $\mathrm{TS} 4 \cdots \mathrm{H}_{2} \mathrm{O}, 26$ & -337.378184 & -337.010856 \\
\hline $\mathrm{N}_{2} \mathrm{O}+\mathrm{H}_{2} \mathrm{O} \cdots \mathrm{H}_{2} \mathrm{O}$ & -337.482212 & -337.119082 \\
\hline \multicolumn{3}{|c|}{$\mathrm{NH}_{2} \mathrm{NO}_{2}+\mathrm{HNO}_{3} \longrightarrow \mathrm{N}_{2} \mathrm{O}+\mathrm{H}_{2} \mathrm{O}+\mathrm{HNO}_{3}$ (Figure 8) } \\
\hline $\mathrm{NH}_{2} \mathrm{NO}_{2} \cdots \mathrm{HNO}_{3}, 4$ & -541.890766 & -541.266258 \\
\hline $\mathrm{TS} 1 \cdots \mathrm{HNO}_{3}, 5$ & -541.878868 & -541.244116 \\
\hline $\mathrm{HNNOOH} \cdots \mathrm{HNO}_{3}, 6$ & -541.879555 & -541.254572 \\
\hline $\mathrm{HNNOOH} \cdots \mathrm{HNO}_{3}, 10$ & -541.868300 & -541.243921 \\
\hline $\mathrm{TS} 2 \cdots \mathrm{HNO}_{3}, 11$ & -541.849545 & -541.217512 \\
\hline $\mathrm{HNNOOH} \cdots \mathrm{HNO}_{3}, 12$ & -541.868571 & -541.244294 \\
\hline $\mathrm{HNNOOH} \cdots \mathrm{HNO}_{3}, 19$ & -541.878994 & -541.254110 \\
\hline $\mathrm{TS} 3 \cdots \mathrm{HNO}_{3}, 20$ & -541.875417 & -541.240147 \\
\hline $\mathrm{HNNOOH} \cdots \mathrm{HNO}_{3}, 21$ & -541.878341 & -541.253413 \\
\hline $\mathrm{HNNOOH} \cdots \mathrm{HNO}_{3}, 27$ & -541.867007 & -541.244036 \\
\hline $\mathrm{N}_{2} \mathrm{O}+\mathrm{H}_{2} \mathrm{O} \cdots \mathrm{HNO}_{3}$ & -541.951751 & -541.326761 \\
\hline \multicolumn{3}{|c|}{$\mathrm{NH}_{2} \mathrm{NO}_{2}+\mathrm{HCOOH} \longrightarrow \mathrm{N}_{2} \mathrm{O}+\mathrm{H}_{2} \mathrm{O}+\mathrm{HCOOH}$ (Figure 8) } \\
\hline $\mathrm{NH}_{2} \mathrm{NO}_{2} \cdots \mathrm{HCOOH}, 7$ & -450.758382 & -450.242701 \\
\hline TS1 $\cdots \mathrm{HCOOH}, 8$ & -450.748195 & -450.220907 \\
\hline $\mathrm{HNNOOH} \cdots \mathrm{HCOOH}, 9$ & -450.748379 & -450.231952 \\
\hline HNNOOH $\cdots$ HCOOH, 13 & -450.736231 & -450.220585 \\
\hline $\mathrm{TS} 2 \cdots \mathrm{HCOOH}, 14$ & -450.720799 & -450.198469 \\
\hline $\mathrm{HNNOOH}^{\cdots} \cdot \mathrm{HCOOH}, 15$ & -450.736472 & -450.220824 \\
\hline HNNOOH $\cdots$ HCOOH, 22 & -450.747937 & -450.231638 \\
\hline TS3 $\cdots \mathrm{HCOOH}, 23$ & -450.744982 & -450.218588 \\
\hline HNNOOH $\cdots$ HCOOH, 24 & -450.747592 & -450.231175 \\
\hline $\mathrm{HNNOOH}^{\cdots} \mathrm{HCOOH}, 28$ & -450.734162 & -450.219678 \\
\hline $\mathrm{N}_{2} \mathrm{O}+\mathrm{H}_{2} \mathrm{O} \cdots \mathrm{HCOOH}$ & -450.812049 & -450.297576 \\
\hline
\end{tabular}


Table S2. Vibrational Frequencies $\left(\mathrm{cm}^{-1}\right)$ and Zero-Point Energy Corrections (ZPE, $\left.\mathrm{kcal} / \mathrm{mol}\right)$ of All Reactants, Transition States, Intermediates and Products Involved in the $\mathrm{H}_{2} \mathrm{NNO} / \mathrm{H}_{2} \mathrm{NNO}_{2}$ Dissociation Pathway

\begin{tabular}{|c|c|c|}
\hline Species/Complexes & Vibrational Frequencies & $\mathrm{ZPE}$ \\
\hline \multicolumn{3}{|l|}{ Single molecules } \\
\hline $\mathrm{N}_{2}$ & 2523 & 3.6 \\
\hline $\mathrm{N}_{2} \mathrm{O}$ & $2422,1359,652,652$ & 7.3 \\
\hline $\mathrm{H}_{2} \mathrm{O}$ & $3971,3869,1620$ & 13.5 \\
\hline $\mathrm{HNO}_{3}$ & $3781,1809,1416,1359,983,825,706,622,506$ & 17.2 \\
\hline cis- $\mathrm{HCOOH}$ & $3867,3025,1911,1431,1289,1141,1057,674,513$ & 21.3 \\
\hline trans-HCOOH & $3791,3099,18691415,1317,1162,1076,674,645$ & 21.5 \\
\hline $\mathrm{NH}_{3}$ & $3635,3635,3510,1660,1660,1032$ & 21.6 \\
\hline HONO & $3662,1783,1373,970,703,687$ & 13.1 \\
\hline \multicolumn{3}{|c|}{$\mathrm{NH}_{2} \mathrm{NO} \longrightarrow \mathrm{N}_{2}+\mathrm{H}_{2} \mathrm{O}$ (Figure 2) } \\
\hline $\mathrm{NH}_{2} \mathrm{NO}, 1$ & $3739,3514,1660,1588,1244,1143,713,656,184$ & 20.6 \\
\hline $\mathrm{TS} 1,2$ & $3553,2158,1514,1395,1240,1182,976,617,1928 \mathrm{i}$ & 18.1 \\
\hline $\mathrm{HNNOH}, 3$ & $3667,3452,1714,1460,1390,1010,982,695,642$ & 21.5 \\
\hline $\mathrm{TS} 2,4$ & $3824,3388,1737,1422,1300,968,909,691,538 \mathrm{i}$ & 20.4 \\
\hline $\mathrm{HNNOH}, 5$ & $3863,3441,1752,1455,1350,993,975,688,495$ & 21.5 \\
\hline $\mathrm{TS} 3,6$ & $3859,3844,1896,1273,704,563,557,474,1287 \mathrm{i}$ & 18.8 \\
\hline $\mathrm{HNNOH}, 7$ & $3853,3280,1780,1431,1323,1035,898,659,471$ & 21.1 \\
\hline $\mathrm{TS} 4,8$ & $3799,2221,1994,1090,1025,789,565,155,1348 \mathrm{i}$ & 16.6 \\
\hline \multicolumn{3}{|c|}{$\mathrm{NH}_{2} \mathrm{NO}+\mathrm{H}_{2} \mathrm{O} \longrightarrow \mathrm{N}_{2}+2 \mathrm{H}_{2} \mathrm{O}$ (Figure 4) } \\
\hline $\mathrm{NH}_{2} \mathrm{NO} \cdots \mathrm{H}_{2} \mathrm{O}, 1$ & $\begin{array}{l}3941,3734,3714,3407,1641,1624,1591,1289,1198,794,695 \\
545,450,366,198,183,141,126\end{array}$ & 36.6 \\
\hline $\mathrm{TS} 1 \cdots \mathrm{H}_{2} \mathrm{O}, 2$ & $\begin{array}{l}3888,3589,2022,1711,1542,1487,1451,1351,1269,1175,864, \\
816,622,605,565,491,280,1616 \mathrm{i}\end{array}$ & 33.9 \\
\hline $\mathrm{HNNOH} \cdots \mathrm{H}_{2} \mathrm{O}, 3$ & $\begin{array}{l}3934,3771,3462,3436,1688,1608,1514,1448,1059,972,820, \\
724,546,309,230,189,176,152\end{array}$ & 37.2 \\
\hline $\mathrm{HNNOH} \cdots \mathrm{H}_{2} \mathrm{O}, 16$ & $\begin{array}{l}3939,3855,3810,3456,1757,1646,1454,1357,990,983,701, \\
520,472,256,147,109,70,54\end{array}$ & 36.6 \\
\hline $\mathrm{TS} 3 \cdots \mathrm{H}_{2} \mathrm{O}, 17$ & $\begin{array}{l}3853,3852,2583,1878,1831,1549,1346,1284,1231,782,652, \\
552,472,416,373,177,109,1304 \mathrm{i}\end{array}$ & 32.8 \\
\hline $\mathrm{HNNOH} \cdots \mathrm{H}_{2} \mathrm{O}, 18$ & $\begin{array}{l}3946,3846,3786,3299,1778,1638,1437,1330,1031,935,671, \\
502,488,312,135,51,38,31\end{array}$ & 36.1 \\
\hline $\mathrm{HNNOH} \cdots \mathrm{H}_{2} \mathrm{O}, 25$ & $\begin{array}{l}3950,3862,3826,3269,1797,1610,1434,1308,1075,809,658, \\
457,439,267,167,153,131,84\end{array}$ & 36.2 \\
\hline $\mathrm{TS} 4 \cdots \mathrm{H}_{2} \mathrm{O}, 26$ & $\begin{array}{l}3904,3897,2932,2018,1786,1574,1317,1266,1029,881,594, \\
534,453,407,387,306,220,633 i\end{array}$ & 33.6 \\
\hline $\mathrm{N}_{2}+\mathrm{H}_{2} \mathrm{O} \cdots \mathrm{H}_{2} \mathrm{O}$ & $\begin{array}{l}3950,3943,3849,3757,2531,1637,1620,621,429,243,191,185 \text {, } \\
167,119,110,86,64,57\end{array}$ & 33.7 \\
\hline \multicolumn{3}{|c|}{$\mathrm{NH}_{2} \mathrm{NO}+\mathrm{HNO}_{3} \longrightarrow \mathrm{N}_{2}+\mathrm{H}_{2} \mathrm{O}+\mathrm{HNO}_{3}$ (Figure 4) } \\
\hline $\mathrm{NH}_{2} \mathrm{NO} \cdots \mathrm{HNO}_{3}, 4$ & $\begin{array}{l}3703,3409,3089,1784,1626,1590,1496,1405,1311,1222,1045, \\
896,834,806,732,689,668,530,217,168,160,145,71,34\end{array}$ & 39.5 \\
\hline $\mathrm{TS} 1 \cdots \mathrm{HNO}_{3}, 5$ & $\begin{array}{l}3584,2004,1808,1700,1675,1620,1522,1434,1234,1231,1189, \\
1118,854,850,829,771,738,681,377,311,201,70,41,499 \mathrm{i}\end{array}$ & 36.9 \\
\hline $\mathrm{HNNOH} \cdots \mathrm{HNO}_{3}, 6$ & $\begin{array}{l}3504,3476,3020,1792,1685,1532,1505,1449,1395,1093,1040, \\
966,912,833,777,733,728,670,215,212,180,142,69,39\end{array}$ & 40.0 \\
\hline $\mathrm{HNNOH} \cdots \mathrm{HNO}_{3}, 10$ & $\begin{array}{l}3661,3537,3448,1798,1730,1465,1440,1408,1352,1015,986, \\
973,832,720,704,698,649,628,190,133,108,65,57,9\end{array}$ & 39.5 \\
\hline $\mathrm{TS} 2 \cdots \mathrm{HNO}_{3}, 11$ & $\begin{array}{l}3421,2119,1776,1739,1712,1457,1420,1374,1260,1216,1089 \\
1010,929,845,804,786,717,482,441,174,126,111,61,1402 \mathrm{i}\end{array}$ & 35.8 \\
\hline $\mathrm{HNNOH} \cdots \mathrm{HNO}_{3}, 12$ & $\begin{array}{l}3832,3510,3442,1794,1759,1462,1434,1406,1309,1016,999 \\
950,832,720,712,702,651,545,197,127,81,69,52,12\end{array}$ & 39.5 \\
\hline $\mathrm{HNNOH} \cdots \mathrm{HNO}_{3}, 19$ & $\begin{array}{l}3850,3468,3219,1791,1759,1489,1441,1404,1360,1020,1011, \\
997,854,835,721,720,657,540,210,157,119,67,49,32\end{array}$ & 39.7 \\
\hline $\mathrm{TS} 3 \cdots \mathrm{HNO}_{3}, 20$ & $\begin{array}{l}3824,2615,2514,1825,1651,1423,1361,1314,1278,1099,1060 \\
1052,862,769,764,736,568,372,311,185,176,122,58,819 \mathrm{i}\end{array}$ & 37.1 \\
\hline
\end{tabular}




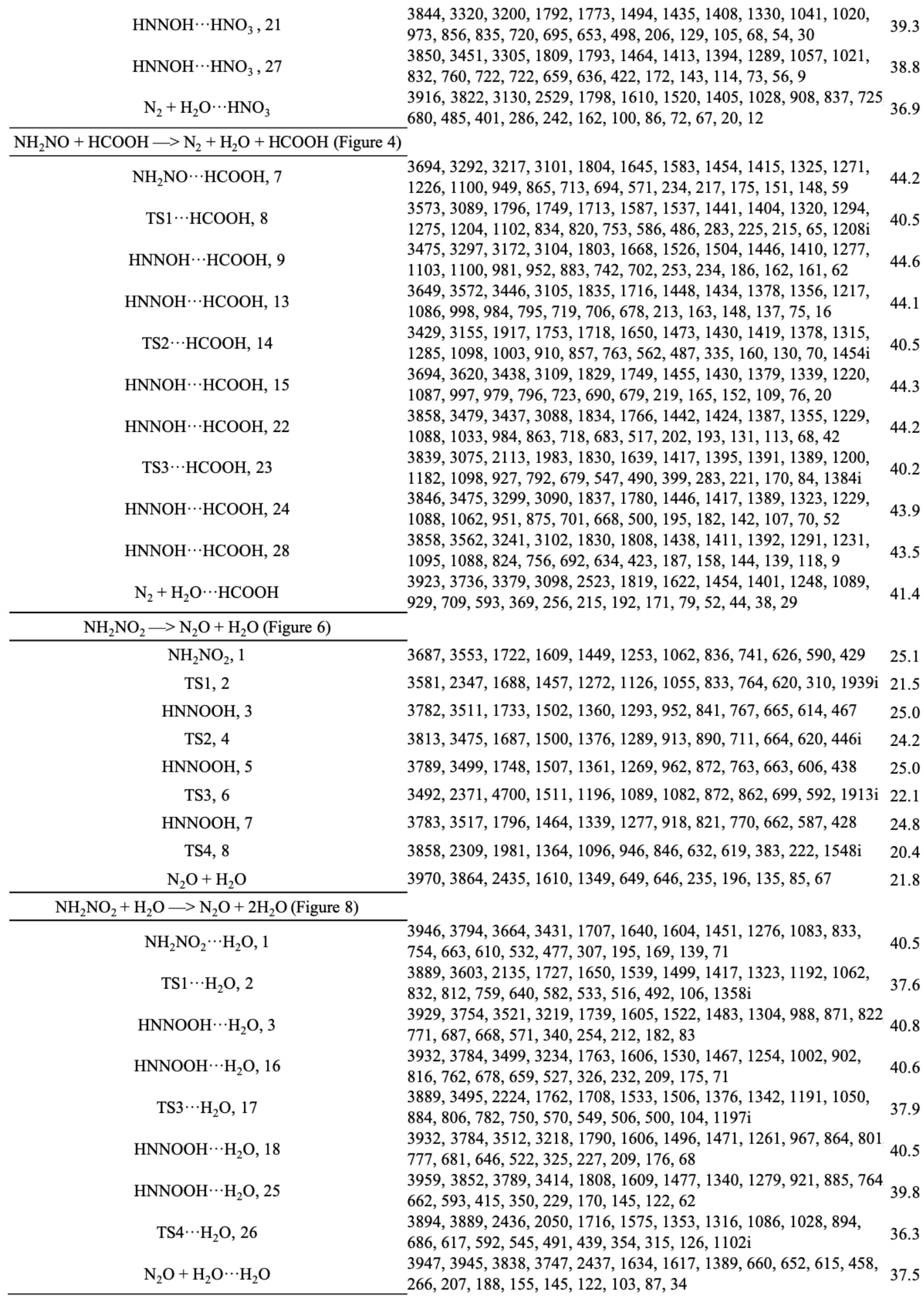




\begin{tabular}{|c|c|c|}
\hline $\mathrm{NH}_{2} \mathrm{NO}_{2}+\mathrm{HNO}_{3} \longrightarrow \mathrm{N}_{2} \mathrm{O}+\mathrm{H}_{2} \mathrm{O}+\mathrm{HNO}_{3}$ (Figure 8) & & \\
\hline $\mathrm{NH}_{2} \mathrm{NO}_{2} \cdots \mathrm{HNO}_{3}, 4$ & $\begin{array}{l}3664,3466,3298,1792,1703,1624,1483,1451,1391,1261,1095, \\
1038,836,828,810,747,728,660,637,598,524,176,152,139,87,56 \\
35\end{array}$ & 43.3 \\
\hline $\mathrm{TS} 1 \cdots \mathrm{HNO}_{3}, 5$ & $\begin{array}{l}3599,1982,1796,1735,1685,1596,1565,1432,1288,1240,1171, \\
1118,1060,852,829,811,749,733,689,641,414,371,212,111,80 \text {, } \\
69,812 \mathrm{i}\end{array}$ & 39.8 \\
\hline $\mathrm{HNNOOH} \cdots \mathrm{HNO}_{3}, 6$ & $\begin{array}{l}3533,3372,2908,1797,1739,1555,1525,1453,1387,1322,1055 \\
1004,924,834,830,765,740,716,695,681,651,202,192,150,109, \\
58,25\end{array}$ & 43.2 \\
\hline $\mathrm{HNNOOH} \cdots \mathrm{HNO}_{3}, 10$ & $\begin{array}{l}3744,3627,3505,1799,1732,1491,1440,1397,1312,1280,1009,935 \\
845,830,763,719,671,653,638,613,523,169,120,65,54,44,16 \\
3493,1965,1755,1712,1635,1606,1426,1413,1387,1246,1244,\end{array}$ & 42.9 \\
\hline $\mathrm{TS} 2 \cdots \mathrm{HNO}_{3}, 11$ & $\begin{array}{l}1069,905,874,934,830,818,758,685,666,466,443,149,108,67,34 \\
1308 \mathrm{i}\end{array}$ & 39.4 \\
\hline $\mathrm{HNNOOH} \cdots \mathrm{HNO}_{3}, 12$ & $\begin{array}{l}3757,3630,3502,1799,1743,1495,1439,1397,1319,1273,1009,946 \\
875,830,763,718,669,649,639,611,495,168,119,65,53,45,18 \\
3497,3408,3159,1800,1755,1529,1509,1441,1390,1233,1051,\end{array}$ & 42.9 \\
\hline $\mathrm{HNNOOH} \cdots \mathrm{HNO}_{3}, 19$ & $\begin{array}{l}1019,903,841,824,762,738,679,671,664,637,189,180,140,90,53 \\
4\end{array}$ & 43.1 \\
\hline $\mathrm{TS} 3 \cdots \mathrm{HNO}_{3}, 20$ & $\begin{array}{l}3488,1839,1767,1655,1628,1593,1498,1459,1287,1243,1231, \\
1117,1054,901,856,841,760,753,727,665,417,401,219,104,99, \\
66,890 \mathrm{i}\end{array}$ & 39.6 \\
\hline $\mathrm{HNNOOH} \cdots \mathrm{HNO}_{3}, 21$ & $\begin{array}{l}3501,3394,3182,1801,1775,1508,1482,1456,1387,1241,1051,993 \\
861,840,821,764,738,685,681,665,623,188,179,136,88,52,1\end{array}$ & 43.0 \\
\hline $\mathrm{HNNOOH} \cdots \mathrm{HNO}_{3}, 27$ & $\begin{array}{l}3773,3581,3476,1825,1797,1453,1439,1400,1325,1279,1013,874 \\
850,829,758,720,675,645,644,582,418,144,127,114,69,46,22\end{array}$ & 42.7 \\
\hline $\mathrm{N}_{2} \mathrm{O}+\mathrm{H}_{2} \mathrm{O} \cdots \mathrm{HNO}_{3}$ & $\begin{array}{l}3944,3826,3230,2426,1798,1594,1494,1404,1361,1018,858,834, \\
722,670,654,651,465,316,241,220,153,111,109,79,51,46,32\end{array}$ & 40.5 \\
\hline \multicolumn{3}{|l|}{$\mathrm{NH}_{2} \mathrm{NO}_{2}+\mathrm{HCOOH} \longrightarrow \mathrm{N}_{2} \mathrm{O}+\mathrm{H}_{2} \mathrm{O}+\mathrm{HCOOH}$ (Figure 8) } \\
\hline $\mathrm{NH}_{2} \mathrm{NO}_{2} \cdots \mathrm{HCOOH}, 7$ & $\begin{array}{l}3658,3457,3297,3111,1810,1692,1641,1454,1437,1403,1273, \\
1257,1099,1095,878,830,765,703,691,609,522,208,190,163,136 \\
74,43\end{array}$ & 47.9 \\
\hline TS1 $\cdots \mathrm{HCOOH}, 8$ & $\begin{array}{l}3591,3129,1791,1759,1689,1627,1544,1455,1429,1399,1288, \\
1227,1155,1099,1091,824,786,783,723,658,461,456,278,219,81 \\
23,1215 \mathrm{i}\end{array}$ & 43.7 \\
\hline HNNOOH $\cdots \mathrm{HCOOH}, 9$ & $\begin{array}{l}3529,3134,3081,2925,1785,1717,1547,1490,1474,1419,1333, \\
1291,1106,1018,997,929,819,755,731,700,660,254,230,201,174 \\
78,49\end{array}$ & 47.8 \\
\hline HNNOOH $\cdots$ HCOOH, 13 & $\begin{array}{l}3690,3505,3464,3120,1826,1717,1504,1427,1372,1358,1285, \\
1209,1087,957,858,789,766,721,684,665,634,212,163,132,72, \\
54,26\end{array}$ & 47.6 \\
\hline $\mathrm{TS} 2 \cdots \mathrm{HCOOH}, 14$ & $\begin{array}{l}3497,3191,2097,1818,1712,1693,1622,1478,1393,1386,1374, \\
1274,1257,1099,969,869,821,786,774,697,649,429,333,111,73 \text {, } \\
30,716 \mathrm{i}\end{array}$ & 44.9 \\
\hline HNNOOH $\cdots$ HCOOH, 15 & $\begin{array}{l}3693,3503,3485,3120,1828,1730,1506,1428,1367,1353,1276 \\
1209,1087,968,882,778,764,695,684,662,630,213,159,128,70 \\
54,23\end{array}$ & 47.6 \\
\hline HNNOOH $\cdots$ HCOOH, 22 & $\begin{array}{l}3502,3339,3125,3020,1792,1749,1530,1469,1449,1415,1279, \\
1238,1100,1030,942,911,839,760,706,680,651,242,209,188,153 \\
66,29\end{array}$ & 47.8 \\
\hline TS3 $\cdots$ HCOOH, 23 & $\begin{array}{l}3497,3148,1791,1769,1681,1635,1535,1488,1408,1386,1303, \\
1260,1238,1100,1082,884,781,773,759,715,503,445,298,208,64 \\
55,988 \mathrm{i}\end{array}$ & 44.0 \\
\hline HNNOOH $\cdots$ HCOOH, 24 & $\begin{array}{l}3505,3352,3126,2989,1796,1760,1502,1482,1444,1413,1279, \\
1247,1101,1005,928,896,820,771,704,688,638,245,208,188,151 \\
63,28\end{array}$ & 47.6 \\
\hline HNNOOH $\cdots$ HCOOH, 28 & $\begin{array}{l}3780,3662,3388,3110,1840,1815,1450,1429,1381,1332,1275, \\
1218,1087,931,855,769,758,680,649,586,420,165,147,138,120 \text {, } \\
72,21\end{array}$ & 47.3 \\
\hline $\mathrm{N}_{2} \mathrm{O}+\mathrm{H}_{2} \mathrm{O} \cdots \mathrm{HCOOH}$ & $\begin{array}{l}3947,3766,3692,3109,2448,1840,1635,1426,1402,1352,1206, \\
1088,758,666,660,655,530,354,198,176,149,144,130,103,42,39 \\
24\end{array}$ & 45.1 \\
\hline
\end{tabular}


Table S3. Relative Energies $(\mathrm{kcal} / \mathrm{mol})$ of All the Reactants, Transition States, Intermediates and Products of $\mathrm{H}_{2} \mathrm{NNO} / \mathrm{H}_{2} \mathrm{NNO}_{2}$ with Water, Formic Acid and Nitric Acid at M06-2X or CCSD(T) Level with aug-cc-pVTZ Basis Set ${ }^{\mathrm{a}}$ 


\begin{tabular}{|c|c|c|c|}
\hline \multirow[b]{2}{*}{ Species/Complexes } & M06-2X & $\operatorname{CCSD}(\mathrm{T})$ & $\operatorname{CCSD}(\mathrm{T})$ \\
\hline & $\begin{array}{l}\text { aug-cc-pVTZ } \\
\text { with ZPE }\end{array}$ & aug-cc-pVTZ & $\begin{array}{l}\text { aug-cc-pVTZ } \\
\text { with ZPE }\end{array}$ \\
\hline \multicolumn{4}{|c|}{$\mathrm{NH}_{2} \mathrm{NO} \longrightarrow \mathrm{N}_{2}+\mathrm{H}_{2} \mathrm{O}$ (Figure 2) } \\
\hline NH2NO, 1 & 0.0 & 0.0 & 0.0 \\
\hline TS1, 2 & 32.7 & 33.4 & 30.8 \\
\hline $\mathrm{HNNOH}, 3$ & 0.2 & -1.1 & -0.3 \\
\hline TS2, 4 & 9.1 & 8.6 & 8.3 \\
\hline HNNOH, 5 & 1.2 & -0.4 & 0.4 \\
\hline TS3, 6 & 36.1 & 37.7 & 35.9 \\
\hline $\mathrm{HNNOH}, 7$ & 1.3 & -0.4 & 0.0 \\
\hline TS4, 8 & 26.9 & 25.4 & 21.4 \\
\hline $\mathrm{N}_{2}+\mathrm{H}_{2} \mathrm{O}$ & -76.1 & -76.5 & -79.4 \\
\hline \multicolumn{4}{|c|}{$\mathrm{NH}_{2} \mathrm{NO}+\mathrm{H}_{2} \mathrm{O} \longrightarrow \mathrm{N}_{2}+2 \mathrm{H}_{2} \mathrm{O}$ (Figure 4) } \\
\hline $\mathrm{NH}_{2} \mathrm{NO} \cdots \mathrm{H}_{2} \mathrm{O}, 1$ & -6.4 & -8.6 & -6.1 \\
\hline $\mathrm{TS} 1 \cdots \mathrm{H}_{2} \mathrm{O}, 2$ & 7.4 & 9.6 & 9.4 \\
\hline $\mathrm{HNNOH} \cdots \mathrm{H}_{2} \mathrm{O}, 3$ & -5.7 & -8.8 & -5.8 \\
\hline $\mathrm{HNNOH} \cdots \mathrm{H}_{2} \mathrm{O}, 16$ & -2.1 & -5.3 & -2.9 \\
\hline $\mathrm{TS} 3 \cdots \mathrm{H}_{2} \mathrm{O}, 17$ & 48.7 & 51.3 & 49.9 \\
\hline $\mathrm{HNNOH} \cdots \mathrm{H}_{2} \mathrm{O}, 18$ & -1.3 & -4.9 & -3.0 \\
\hline $\mathrm{HNNOH} \cdots \mathrm{H}_{2} \mathrm{O}, 25$ & -2.5 & -6.0 & -4.1 \\
\hline $\mathrm{TS} 4 \cdots \mathrm{H}_{2} \mathrm{O}, 26$ & 3.8 & 1.6 & 1.0 \\
\hline $\mathrm{N}_{2}+\mathrm{H}_{2} \mathrm{O} \cdots \mathrm{H}_{2} \mathrm{O}$ & -79.8 & -82.5 & -83.0 \\
\hline \multicolumn{4}{|c|}{$\mathrm{NH}_{2} \mathrm{NO}+\mathrm{HNO}_{3} \longrightarrow \mathrm{N}_{2}+\mathrm{H}_{2} \mathrm{O}+\mathrm{HNO}_{3}$ (Figure 4) } \\
\hline $\mathrm{NH}_{2} \mathrm{NO}_{\cdots} \mathrm{HNO}_{3}, 4$ & -11.2 & -12.7 & -11.0 \\
\hline $\mathrm{TS} 1 \cdots \mathrm{HNO}_{3}, 5$ & -6.1 & -3.1 & -4.0 \\
\hline $\mathrm{HNNOH} \cdots \mathrm{HNO}_{3}, 6$ & -9.9 & -12.2 & -10.1 \\
\hline $\mathrm{HNNOH} \cdots \mathrm{HNO}_{3}, 10$ & -6.9 & -8.9 & -7.2 \\
\hline $\mathrm{TS} 2 \cdots \mathrm{HNO}_{3}, 11$ & 6.4 & 9.5 & 7.5 \\
\hline $\mathrm{HNNOH} \cdots \mathrm{HNO}_{3}, 12$ & -6.7 & -8.9 & -7.3 \\
\hline $\mathrm{HNNOH} \cdots \mathrm{HNO}_{3}, 19$ & -7.9 & -10.3 & -8.4 \\
\hline $\mathrm{TS} 3 \cdots \mathrm{HNO}_{3}, 20$ & 10.3 & 11.8 & 11.1 \\
\hline $\mathrm{HNNOH} \cdots \mathrm{HNO}_{3}, 21$ & -7.3 & -9.8 & -8.3 \\
\hline $\mathrm{HNNOH} \cdots \mathrm{HNO}_{3}, 27$ & -5.6 & -8.7 & -7.7 \\
\hline $\mathrm{N}_{2}+\mathrm{H}_{2} \mathrm{O} \cdots \mathrm{HNO}_{3}$ & -86.1 & -87.7 & -88.6 \\
\hline \multicolumn{4}{|c|}{$\mathrm{NH}_{2} \mathrm{NO}+\mathrm{HCOOH} \longrightarrow \mathrm{N}_{2}+\mathrm{H}_{2} \mathrm{O}+\mathrm{HCOOH}$ (Figure 4) } \\
\hline $\mathrm{NH}_{2} \mathrm{NO} \cdots \mathrm{HCOOH}, 7$ & -16.0 & -18.2 & -16.0 \\
\hline TS1 $\cdots \mathrm{HCOOH}, 8$ & -11.2 & -7.6 & -9.0 \\
\hline $\mathrm{HNNOH} \cdots \mathrm{HCOOH}, 9$ & -14.9 & -17.8 & -15.2 \\
\hline HNNOH $\cdots \mathrm{HCOOH}, 13$ & -11.1 & -13.4 & -11.3 \\
\hline $\mathrm{TS} 2 \cdots \mathrm{HCOOH}, 14$ & 1.0 & 3.7 & 2.2 \\
\hline $\mathrm{HNNOH} \cdots \mathrm{HCOOH}, 15$ & -11.3 & -14.0 & -11.7 \\
\hline HNNOH $\cdots \mathrm{HCOOH}, 22$ & -10.7 & -13.6 & -11.3 \\
\hline TS3 $\cdots \mathrm{HCOOH}, 23$ & 8.0 & 10.4 & 8.7 \\
\hline HNNOH $\cdots \mathrm{HCOOH}, 24$ & -9.9 & -13.0 & -11.0 \\
\hline HNNOH $\cdots \mathrm{HCOOH}, 28$ & -10.1 & -13.7 & -12.2 \\
\hline $\mathrm{N}_{2}+\mathrm{H}_{2} \mathrm{O} \cdots \mathrm{HCOOH}$ & -89.6 & -91.4 & -91.9 \\
\hline
\end{tabular}




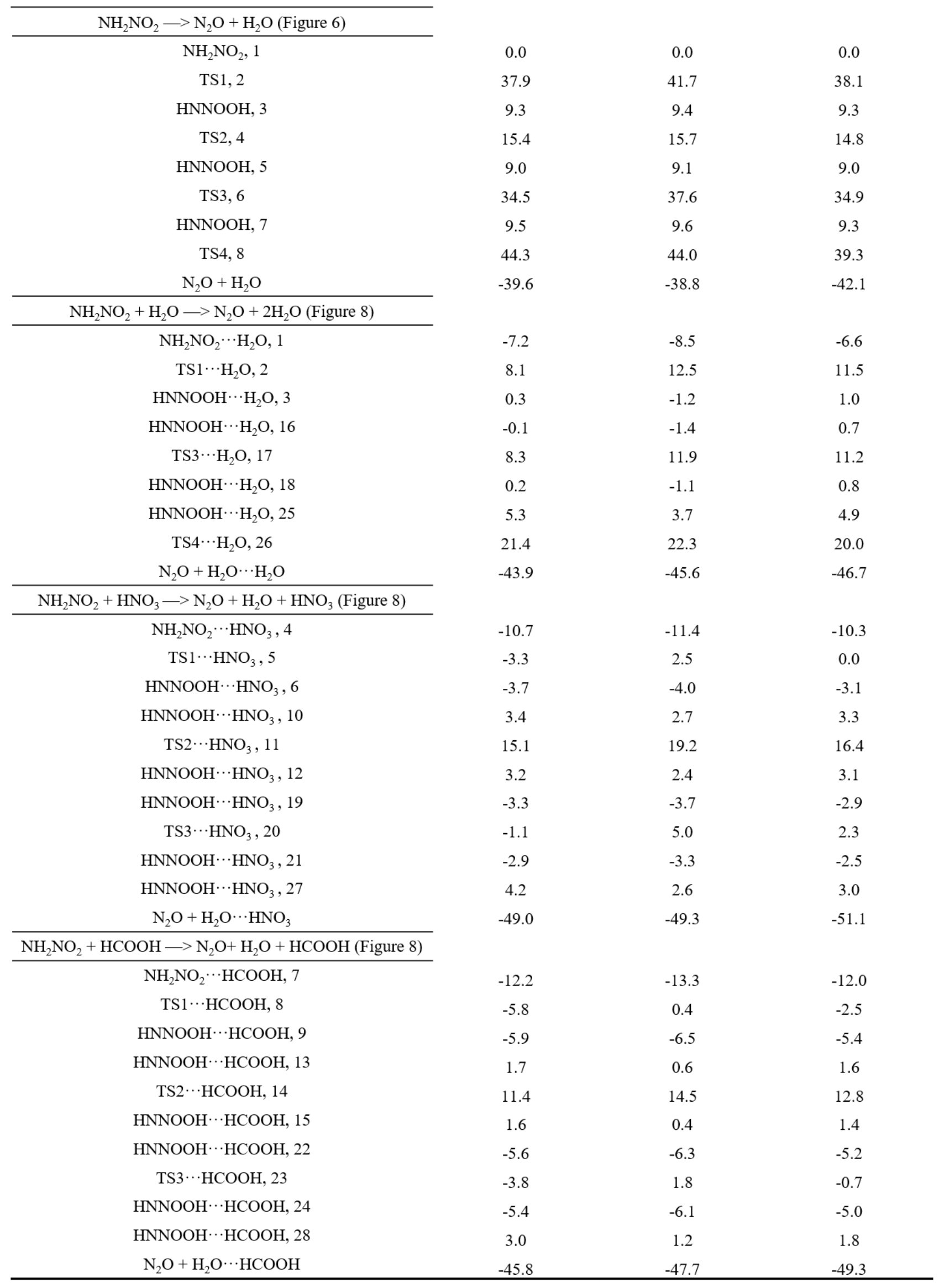

${ }^{a}$ The corrected ZPE from M06-2X/aug-cc-pVTZ are included. 
Table S4. Binding Energies of All Related Complexes at M06-2X and CCSD(T) levels of Theories with aug-cc-pVTZ basis set. Zero-Point Vibrational Energy (ZPE) from M06-2X/aug-cc-pVTZ are Included for Corrections

\begin{tabular}{|c|c|c|}
\hline \multirow{2}{*}{ Complexes } & M06-2X & $\operatorname{CCSD}(\mathrm{T})$ \\
\hline & aug-cc-pVTZ & aug-cc-pVTZ \\
\hline \multicolumn{3}{|l|}{ Products } \\
\hline $\mathrm{H}_{2} \mathrm{O} \cdots \mathrm{H}_{2} \mathrm{O}$ & 3.2 & 3.2 \\
\hline $\mathrm{H}_{2} \mathrm{O} \cdots \mathrm{HNO}_{3}$ & 9.5 & 8.5 \\
\hline $\mathrm{H}_{2} \mathrm{O} \cdots \mathrm{HCOOH}$ & 12.9 & 12.2 \\
\hline \multicolumn{3}{|l|}{ Figure 4} \\
\hline $\mathrm{NH}_{2} \mathrm{NO} \cdots \mathrm{H}_{2} \mathrm{O}, 1$ & 6.4 & 6.1 \\
\hline $\mathrm{HNNOH} \cdots \mathrm{H}_{2} \mathrm{O}, 3$ & 5.9 & 5.5 \\
\hline $\mathrm{HNNOH} \cdots \mathrm{H}_{2} \mathrm{O}, 16$ & 3.2 & 3.3 \\
\hline $\mathrm{HNNOH} \cdots \mathrm{H}_{2} \mathrm{O}, 18$ & 2.7 & 3.0 \\
\hline $\mathrm{HNNOH} \cdots \mathrm{H}_{2} \mathrm{O}, 25$ & 3.8 & 4.0 \\
\hline $\mathrm{NH}_{2} \mathrm{NO} \cdots \mathrm{HNO}_{3}, 4$ & 11.2 & 11.0 \\
\hline $\mathrm{HNNOH} \cdots \mathrm{HNO}_{3}, 6$ & 10.1 & 9.8 \\
\hline $\mathrm{HNNOH} \cdots \mathrm{HNO}_{3}, 10$ & 7.0 & 7.0 \\
\hline $\mathrm{HNNOH} \cdots \mathrm{HNO}_{3}, 12$ & 7.9 & 7.7 \\
\hline $\mathrm{HNNOH} \cdots \mathrm{HNO}_{3}, 19$ & 9.1 & 8.8 \\
\hline $\mathrm{HNNOH} \cdots \mathrm{HNO}_{3}, 21$ & 8.7 & 8.3 \\
\hline $\mathrm{HNNOH} \cdots \mathrm{HNO}_{3}, 27$ & 7.0 & 7.7 \\
\hline $\mathrm{NH}_{2} \mathrm{NO} \cdots \mathrm{HCOOH}, 7$ & 16.0 & 16.0 \\
\hline HNNOH $\cdots \mathrm{HCOOH}, 9$ & 15.1 & 14.9 \\
\hline HNNOH $\cdots \mathrm{HCOOH}, 13$ & 11.2 & 11.0 \\
\hline HNNOH $\cdots \mathrm{HCOOH}, 15$ & 12.5 & 12.1 \\
\hline $\mathrm{HNNOH} \cdots \mathrm{HCOOH}, 22$ & 11.9 & 11.7 \\
\hline $\mathrm{HNNOH} \cdots \mathrm{HCOOH}, 24$ & 11.2 & 11.0 \\
\hline HNNOH $\cdots \mathrm{HCOOH}, 28$ & 11.4 & 12.2 \\
\hline \multicolumn{3}{|l|}{ Figure 8} \\
\hline $\mathrm{NH}_{2} \mathrm{NO}_{2} \cdots \mathrm{H}_{2} \mathrm{O}, 1$ & 7.3 & 6.6 \\
\hline $\mathrm{HNNOOH} \cdots \mathrm{H}_{2} \mathrm{O}, 3$ & 9.0 & 8.4 \\
\hline $\mathrm{HNNOOH} \cdots \mathrm{H}_{2} \mathrm{O}, 16$ & 9.1 & 8.7 \\
\hline $\mathrm{HNNOOH} \cdots \mathrm{H}_{2} \mathrm{O}, 18$ & 9.3 & 8.2 \\
\hline $\mathrm{HNNOOH} \cdots \mathrm{H}_{2} \mathrm{O}, 25$ & 4.2 & 4.1 \\
\hline $\mathrm{NH}_{2} \mathrm{NO}_{2} \cdots \mathrm{HNO}_{3}, 4$ & 10.7 & 10.3 \\
\hline $\mathrm{HNNOOH} \cdots \mathrm{HNO}_{3}, 6$ & 13.0 & 12.4 \\
\hline $\mathrm{HNNOOH} \cdots \mathrm{HNO}_{3}, 10$ & 5.9 & 6.1 \\
\hline $\mathrm{HNNOOH} \cdots \mathrm{HNO}_{3}, 12$ & 5.8 & 5.9 \\
\hline $\mathrm{HNNOOH} \cdots \mathrm{HNO}_{3}, 19$ & 12.3 & 11.9 \\
\hline $\mathrm{HNNOOH} \cdots \mathrm{HNO}_{3}, 21$ & 12.4 & 11.9 \\
\hline $\mathrm{HNNOOH} \cdots \mathrm{HNO}_{3}, 27$ & 5.3 & 6.3 \\
\hline $\mathrm{NH}_{2} \mathrm{NO}_{2} \cdots \mathrm{HCOOH}, 7$ & 16.4 & 16.0 \\
\hline HNNOOH $\cdots \mathrm{HCOOH}, 9$ & 19.4 & 18.7 \\
\hline HNNOOH $\cdots \mathrm{HCOOH}, 13$ & 11.7 & 11.7 \\
\hline HNNOOH $\cdots \mathrm{HCOOH}, 15$ & 11.6 & 11.6 \\
\hline HNNOOH $\cdots \mathrm{HCOOH}, 22$ & 18.8 & 18.2 \\
\hline HNNOOH $\cdots \mathrm{HCOOH}, 24$ & 19.1 & 18.4 \\
\hline HNNOOH $\cdots \mathrm{HCOOH}, 28$ & 10.7 & 11.5 \\
\hline
\end{tabular}


Table S5. Cartesian Coordinates for All Optimized Structures in M06-2X/cc-aug-pVTZ Level 


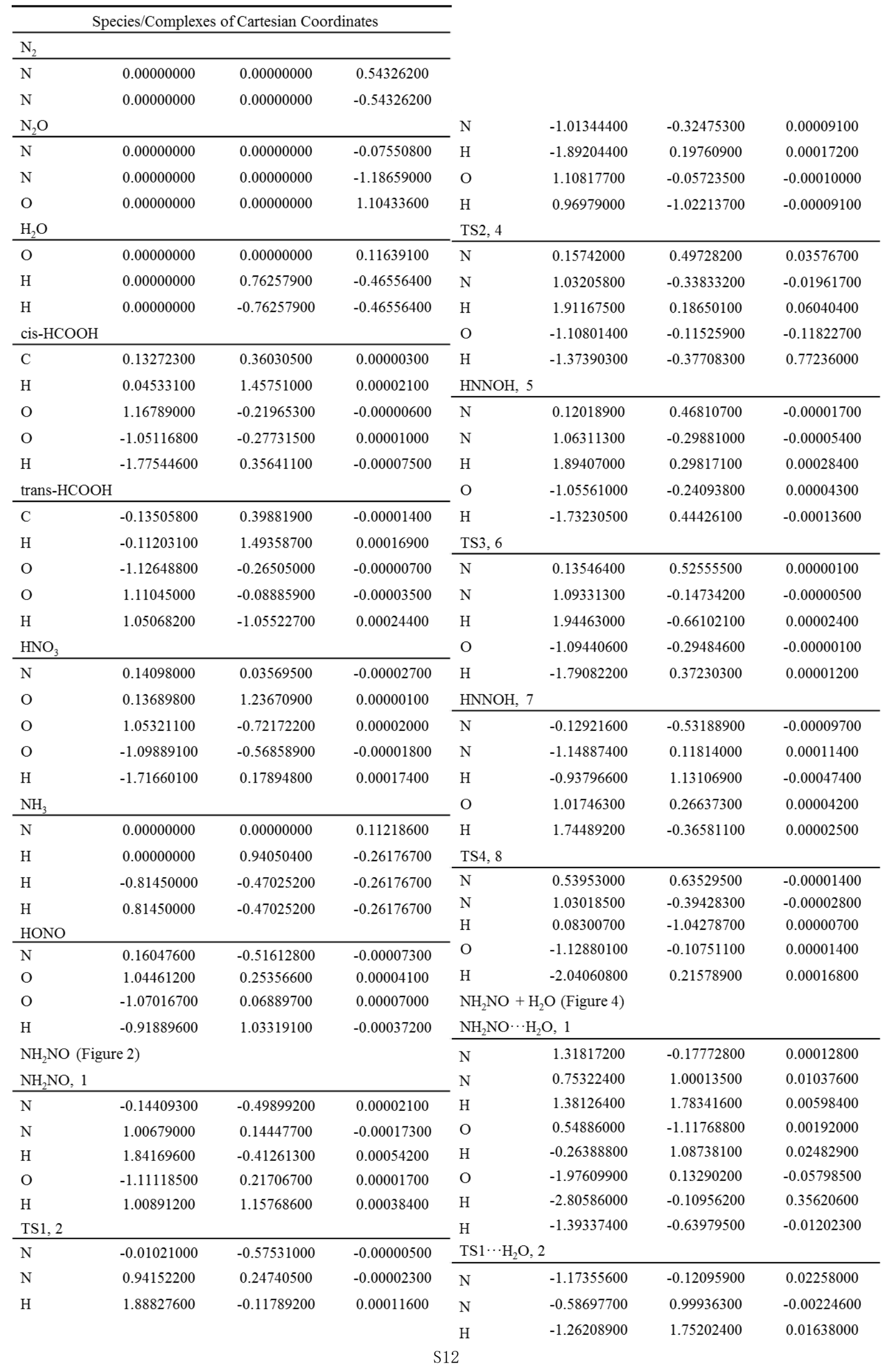




\begin{tabular}{|c|c|c|c|c|c|c|c|}
\hline $\mathrm{O}$ & -0.39611800 & -1.13259700 & -0.00124400 & $\mathrm{O}$ & -0.55306100 & 1.03126100 & -0.00601600 \\
\hline $\mathrm{H}$ & 0.69933500 & 0.86399100 & -0.02288900 & $\mathrm{H}$ & -1.16888100 & 1.77161600 & -0.00510600 \\
\hline $\mathrm{O}$ & 1.63199800 & 0.11076000 & -0.09635400 & $\mathrm{O}$ & 2.05985500 & -0.10759600 & -0.07319700 \\
\hline $\mathrm{H}$ & 2.19977100 & 0.11161300 & 0.67940900 & $\mathrm{H}$ & 1.53949900 & 0.69677700 & 0.02896000 \\
\hline $\mathrm{H}$ & 0.79967000 & -0.70176500 & -0.03445500 & $\mathrm{H}$ & 0.22335300 & -1.01689700 & -0.02344800 \\
\hline \multicolumn{4}{|c|}{$\mathrm{HNNOH} \cdots \mathrm{H}_{2} \mathrm{O}, 3$} & \multicolumn{4}{|c|}{$\mathrm{TS} 4 \cdots \mathrm{H}_{2} \mathrm{O}, 26$} \\
\hline $\mathrm{N}$ & -1.33999300 & -0.04882400 & 0.01392700 & $\mathrm{~N}$ & -1.31009400 & -0.25214100 & -0.01328200 \\
\hline $\mathrm{N}$ & -0.72177700 & 1.01186400 & -0.00070900 & $\mathrm{~N}$ & -0.61842900 & -1.16609500 & 0.01686600 \\
\hline $\mathrm{H}$ & -1.43029200 & 1.74673700 & 0.00668100 & $\mathrm{H}$ & 0.50204800 & -0.95752200 & 0.02557800 \\
\hline $\mathrm{O}$ & -0.55533500 & -1.13382400 & 0.00445200 & $\mathrm{O}$ & -0.35479500 & 1.21802500 & 0.07849400 \\
\hline $\mathrm{H}$ & 1.42684500 & 0.84832300 & 0.00024900 & $\mathrm{H}$ & -0.92127100 & 1.87666600 & -0.33025700 \\
\hline $\mathrm{O}$ & 1.97846600 & 0.05893400 & -0.08788600 & $\mathrm{O}$ & 1.67817000 & -0.15956700 & -0.11241700 \\
\hline $\mathrm{H}$ & 2.66172800 & 0.11403000 & 0.58349100 & $\mathrm{H}$ & 2.24832200 & -0.11786000 & 0.66033500 \\
\hline $\mathrm{H}$ & 0.38906100 & -0.85124900 & -0.01548300 & $\mathrm{H}$ & 1.08356100 & 0.65870500 & -0.10935800 \\
\hline \multicolumn{4}{|c|}{$\mathrm{HNNOH} \cdots \mathrm{H}_{2} \mathrm{O}, 16$} & \multicolumn{4}{|c|}{$\mathrm{N}_{2}+\mathrm{H}_{2} \mathrm{O} \cdots \mathrm{H}_{2} \mathrm{O}$} \\
\hline $\mathrm{N}$ & -1.39222700 & 0.18144200 & -0.00047200 & $\mathrm{~N}$ & -2.27381400 & 0.04669600 & 0.00476100 \\
\hline $\mathrm{N}$ & -0.54336800 & 1.05170600 & 0.00042600 & $\mathrm{~N}$ & -1.39982100 & -0.59720300 & -0.00858900 \\
\hline $\mathrm{H}$ & -1.04979400 & 1.93959400 & -0.00036700 & $\mathrm{H}$ & 0.76947100 & -1.38120300 & -0.05530600 \\
\hline $\mathrm{O}$ & -0.79059900 & -1.04906000 & 0.00027200 & $\mathrm{O}$ & 0.82819400 & 1.59732900 & 0.08037200 \\
\hline $\mathrm{H}$ & -1.52929900 & -1.66765600 & -0.00045900 & $\mathrm{H}$ & 1.30234500 & 2.25341800 & -0.43264000 \\
\hline $\mathrm{O}$ & 2.35222100 & -0.00035400 & -0.00014400 & $\mathrm{O}$ & 1.69672400 & -1.12758700 & -0.09578100 \\
\hline $\mathrm{H}$ & 1.48847900 & 0.42850400 & -0.00014100 & $\mathrm{H}$ & 2.11814500 & -1.55075600 & 0.65547100 \\
\hline $\mathrm{H}$ & 2.14680200 & -0.93717100 & 0.00026900 & $\mathrm{H}$ & 1.32613800 & 0.77415800 & -0.01746100 \\
\hline \multicolumn{4}{|c|}{$\mathrm{TS} 3 \cdots \mathrm{H}_{2} \mathrm{O}, 17$} & \multicolumn{4}{|c|}{$\mathrm{NH}_{2} \mathrm{NO}+\mathrm{HCOOH}$ (Figure 4) } \\
\hline $\mathrm{N}$ & -1.00826900 & 0.62886700 & 0.03972700 & \multicolumn{4}{|c|}{$\mathrm{NH}_{2} \mathrm{NO} \cdots \mathrm{HCOOH}, 7$} \\
\hline $\mathrm{N}$ & 0.12553100 & 0.31042500 & -0.04499700 & $\mathrm{~N}$ & 2.04716400 & -0.21880600 & -0.00001600 \\
\hline $\mathrm{H}$ & 1.60641800 & 0.63683300 & -0.00249800 & $\mathrm{~N}$ & 1.55850600 & 0.97966600 & -0.00008100 \\
\hline $\mathrm{O}$ & -1.89709800 & -0.55060000 & -0.00324900 & $\mathrm{H}$ & 2.23721500 & 1.72108200 & -0.00019600 \\
\hline $\mathrm{H}$ & -2.77116100 & -0.15045800 & 0.06094400 & $\mathrm{O}$ & 1.21856600 & -1.11740400 & 0.00011500 \\
\hline $\mathrm{O}$ & 2.30689200 & -0.20295900 & -0.08215900 & $\mathrm{H}$ & 0.54166900 & 1.13648600 & -0.00000400 \\
\hline $\mathrm{H}$ & 2.75174100 & -0.36710300 & 0.75831800 & $\mathrm{C}$ & -1.96671000 & 0.15075800 & -0.00001700 \\
\hline $\mathrm{H}$ & 1.31381700 & -0.66584800 & -0.09660700 & $\mathrm{H}$ & -3.06126300 & 0.16064300 & -0.00008700 \\
\hline \multicolumn{4}{|c|}{$\mathrm{HNNOH} \cdots \mathrm{H}_{2} \mathrm{O}, 18$} & $\mathrm{O}$ & -1.30726300 & 1.16418100 & 0.00011700 \\
\hline $\mathrm{N}$ & -0.97282600 & -0.46994000 & -0.00123200 & $\mathrm{O}$ & -1.49335700 & -1.07068300 & -0.00009800 \\
\hline $\mathrm{N}$ & -0.08518400 & 0.35395500 & 0.00365800 & $\mathrm{H}$ & -0.50062700 & -1.05753400 & -0.00000500 \\
\hline $\mathrm{H}$ & 1.96148900 & 0.00556900 & -0.00109400 & \multicolumn{4}{|c|}{$\mathrm{TS} 1 \cdots \mathrm{HCOOH}, 8$} \\
\hline $\mathrm{O}$ & -2.23338300 & 0.10579900 & -0.00074000 & $\mathrm{~N}$ & -1.89260800 & -0.01800800 & -0.00004100 \\
\hline $\mathrm{H}$ & -2.83946000 & -0.64404200 & -0.00498000 & $\mathrm{~N}$ & -1.20378800 & 1.03236200 & 0.00004500 \\
\hline $\mathrm{O}$ & 2.92664500 & 0.02285000 & -0.00489800 & $\mathrm{H}$ & -1.80202000 & 1.84935100 & 0.00000400 \\
\hline $\mathrm{H}$ & 3.20070200 & -0.89493700 & 0.02704600 & $\mathrm{O}$ & -1.24293700 & -1.10947700 & -0.00000900 \\
\hline $\mathrm{H}$ & -0.46275700 & 1.31612000 & 0.00715000 & $\mathrm{H}$ & 0.01025000 & 1.08316700 & 0.00003500 \\
\hline \multicolumn{4}{|c|}{$\mathrm{HNNOH} \cdots \mathrm{H}_{2} \mathrm{O}, 25$} & $\mathrm{C}$ & 1.80470400 & -0.02279000 & -0.00002000 \\
\hline $\mathrm{N}$ & -1.40920200 & -0.11136000 & 0.01900500 & $\mathrm{H}$ & 2.89852800 & -0.08254800 & -0.00008300 \\
\hline $\mathrm{N}$ & -0.80363600 & -1.15099700 & 0.00390900 & $\mathrm{O}$ & 1.29243000 & 1.12161500 & -0.00001300 \\
\hline $\mathrm{H}$ & 2.84153800 & -0.00431500 & 0.47289100 & $\mathrm{O}$ & 1.17758900 & -1.10831100 & 0.00003700 \\
\hline
\end{tabular}




\begin{tabular}{|c|c|c|c|c|c|c|c|}
\hline \multirow{2}{*}{\multicolumn{2}{|c|}{$\begin{array}{l}\mathrm{H} \\
\mathrm{HNNOH} \cdots \mathrm{HCOOH}, 9 \\
\end{array}$}} & \multirow{2}{*}{-1.04432600} & \multirow{2}{*}{0.00001500} & \multirow{3}{*}{$\begin{array}{l}\mathrm{O} \\
\mathrm{H} \\
\mathrm{C}\end{array}$} & \multirow{3}{*}{$\begin{array}{l}2.74472800 \\
3.66493800 \\
-2.33732200\end{array}$} & \multirow{3}{*}{$\begin{array}{l}0.56386400 \\
0.27825700 \\
0.12655700\end{array}$} & \multirow{3}{*}{$\begin{array}{l}0.00011700 \\
0.00079300 \\
-0.00001900\end{array}$} \\
\hline & & & & & & & \\
\hline $\mathrm{N}$ & -2.05607400 & 0.11554300 & -0.00001000 & & & & \\
\hline $\mathrm{N}$ & -1.24805700 & 1.04452500 & 0.00038200 & $\mathrm{H}$ & -3.38826300 & 0.43567400 & -0.00041900 \\
\hline $\mathrm{H}$ & -1.79878200 & 1.90318700 & 0.00066900 & $\mathrm{O}$ & -1.98328000 & -1.02190300 & 0.00031600 \\
\hline $\mathrm{O}$ & -1.50743900 & -1.09005300 & -0.00042400 & $\mathrm{O}$ & -1.53686900 & 1.18084400 & 0.00001500 \\
\hline $\mathrm{H}$ & 0.54616100 & 1.10581700 & -0.00016600 & $\mathrm{H}$ & -0.60375000 & 0.87317800 & 0.00021300 \\
\hline $\mathrm{C}$ & 1.95208900 & -0.15167800 & 0.00000400 & \multicolumn{4}{|c|}{$\mathrm{TS} 3 \cdots \mathrm{HCOOH}, 23$} \\
\hline $\mathrm{H}$ & 3.04368900 & -0.22607300 & -0.00011200 & $\mathrm{~N}$ & 1.75919900 & -0.61896900 & 0.00001500 \\
\hline $\mathrm{O}$ & 1.54178600 & 1.09329900 & -0.00040400 & $\mathrm{~N}$ & 0.58942100 & -0.32209100 & -0.00017700 \\
\hline $\mathrm{O}$ & 1.23332700 & -1.12387400 & 0.00046500 & $\mathrm{H}$ & -0.36221900 & -1.01196400 & -0.00012200 \\
\hline $\mathrm{H}$ & -0.51607300 & -1.02831300 & -0.00010900 & $\mathrm{O}$ & 2.55264500 & 0.49207100 & 0.00006400 \\
\hline \multicolumn{4}{|c|}{$\mathrm{HNNOH} \cdots \mathrm{HCOOH}, 13$} & $\mathrm{H}$ & 3.45077600 & 0.13989300 & 0.00026100 \\
\hline $\mathrm{N}$ & 2.13933500 & -0.52247800 & 0.01222000 & $\mathrm{C}$ & -2.07997700 & 0.18929300 & 0.00006900 \\
\hline $\mathrm{N}$ & 2.65304000 & 0.56718600 & -0.16941800 & $\mathrm{H}$ & -3.15765700 & 0.39042900 & 0.00019600 \\
\hline $\mathrm{H}$ & 3.64328300 & 0.38528700 & -0.34911900 & $\mathrm{O}$ & -1.72563000 & -1.02067300 & 0.00004800 \\
\hline $\mathrm{O}$ & 0.80753800 & -0.41889200 & 0.26765700 & $\mathrm{O}$ & -1.31057600 & 1.18521000 & -0.00004000 \\
\hline $\mathrm{H}$ & -0.94628100 & -1.12825800 & -0.02791400 & $\mathrm{H}$ & -0.02288200 & 0.68044700 & -0.00018700 \\
\hline $\mathrm{C}$ & -2.21103000 & 0.27170200 & -0.06481100 & \multicolumn{4}{|c|}{ HNNOH $\cdots$ HCOOH, 24} \\
\hline $\mathrm{H}$ & -3.28957400 & 0.42445000 & -0.16995100 & $\mathrm{~N}$ & 2.01343900 & -0.59708000 & 0.00002900 \\
\hline $\mathrm{O}$ & -1.91107600 & -1.01711200 & -0.12356300 & $\mathrm{~N}$ & 0.84165500 & -0.29206400 & -0.00085500 \\
\hline $\mathrm{O}$ & -1.42382400 & 1.16770500 & 0.08296200 & $\mathrm{H}$ & -0.90822000 & -1.01984300 & -0.00008700 \\
\hline $\mathrm{H}$ & 0.53102300 & 0.52173300 & 0.21978600 & $\mathrm{O}$ & 2.84586100 & 0.50664000 & 0.00037700 \\
\hline \multicolumn{4}{|c|}{$\mathrm{TS} 2 \cdots \mathrm{HCOOH}, 14$} & $\mathrm{H}$ & 3.73531200 & 0.13462200 & 0.00101800 \\
\hline $\mathrm{N}$ & 1.73194400 & -0.52772100 & -0.11955200 & $\mathrm{C}$ & -2.35864600 & 0.18448600 & 0.00021400 \\
\hline $\mathrm{N}$ & 2.29458500 & 0.54808500 & -0.24219200 & $\mathrm{H}$ & -3.45417700 & 0.18708300 & 0.00052300 \\
\hline $\mathrm{H}$ & 3.13831600 & 0.35193500 & -0.78791600 & $\mathrm{O}$ & -1.89040600 & -1.05432000 & 0.00023700 \\
\hline $\mathrm{O}$ & 0.58868900 & -0.40243400 & 0.61496200 & $\mathrm{O}$ & -1.69136100 & 1.18336900 & -0.00009800 \\
\hline $\mathrm{H}$ & -0.42953600 & -0.99895900 & 0.15739300 & $\mathrm{H}$ & 0.68054700 & 0.72971700 & -0.00108300 \\
\hline $\mathrm{C}$ & -1.88006800 & 0.21778700 & -0.20366500 & \multicolumn{4}{|c|}{$\mathrm{HNNOH} \cdots \mathrm{HCOOH}, 28$} \\
\hline $\mathrm{H}$ & -2.89048200 & 0.46534600 & -0.52887200 & $\mathrm{~N}$ & 2.20081000 & -0.01524100 & -0.00830700 \\
\hline $\mathrm{O}$ & -1.53958600 & -0.99740600 & -0.21139900 & $\mathrm{~N}$ & 1.71298200 & -1.10950600 & -0.00894600 \\
\hline $\mathrm{O}$ & -1.12611800 & 1.16078700 & 0.15524000 & $\mathrm{H}$ & -0.64181400 & 1.06947600 & -0.00636500 \\
\hline $\mathrm{H}$ & -0.10747400 & 0.64483100 & 0.44317800 & $\mathrm{O}$ & 1.21045500 & 1.03953700 & 0.01493800 \\
\hline \multicolumn{4}{|c|}{$\mathrm{HNNOH} \cdots \mathrm{HCOOH}, 15$} & $\mathrm{H}$ & 1.73979500 & 1.84447800 & 0.01380000 \\
\hline $\mathrm{N}$ & 2.06644800 & -0.48080500 & -0.12845100 & $\mathrm{C}$ & -2.03477900 & -0.19544400 & -0.00095600 \\
\hline $\mathrm{N}$ & 2.81667400 & 0.47113700 & -0.03804700 & $\mathrm{H}$ & -3.12779900 & -0.25320300 & -0.00486900 \\
\hline $\mathrm{H}$ & 3.72708400 & 0.12592300 & -0.35254600 & $\mathrm{O}$ & -1.62111200 & 1.05882300 & -0.01186600 \\
\hline $\mathrm{O}$ & 0.80726300 & -0.12271300 & 0.27957200 & $\mathrm{O}$ & -1.31856800 & -1.16156700 & 0.01162300 \\
\hline $\mathrm{H}$ & 0.23506500 & -0.89819200 & 0.14306500 & $\mathrm{H}$ & 0.67573700 & -1.10921200 & 0.00637800 \\
\hline $\mathrm{C}$ & -2.25982500 & -0.01042600 & -0.07458500 & \multicolumn{4}{|c|}{$\mathrm{N}_{2}+\mathrm{H}_{2} \mathrm{O} \cdots \mathrm{HCOOH}$ (Figure 4) } \\
\hline $\mathrm{H}$ & -3.34302200 & 0.10285600 & -0.17835900 & $\mathrm{~N}$ & -2.17300100 & -0.87074200 & -0.11961600 \\
\hline $\mathrm{O}$ & -1.70808200 & -1.07882500 & -0.02441100 & $\mathrm{~N}$ & -2.14850200 & 0.20349200 & 0.04022600 \\
\hline $\mathrm{O}$ & -1.66674800 & 1.17008500 & -0.01470500 & $\mathrm{H}$ & 0.51520700 & 1.42320400 & 0.80050300 \\
\hline $\mathrm{H}$ & -0.70150400 & 1.05127400 & 0.07719200 & $\mathrm{O}$ & 0.47741500 & 1.98658100 & 0.01204400 \\
\hline \multicolumn{4}{|c|}{ HNNOH $\cdots \mathrm{HCOOH}, 22$} & $\mathrm{H}$ & -0.39839700 & 2.37957600 & 0.00076900 \\
\hline $\mathrm{N}$ & 2.03935800 & -0.60695200 & 0.00016000 & $\mathrm{C}$ & 1.19546600 & -1.02093800 & 0.05975900 \\
\hline $\mathrm{N}$ & 0.84751800 & -0.37336200 & -0.00066600 & $\mathrm{H}$ & 1.48504100 & -2.06838100 & -0.07236700 \\
\hline $\mathrm{H}$ & 0.34624400 & -1.26669500 & -0.00050700 & $\mathrm{O}$ & 0.96507500 & -0.53106600 & 1.13672500 \\
\hline
\end{tabular}




\begin{tabular}{|c|c|c|c|c|c|c|c|}
\hline $\mathrm{O}$ & 1.13213800 & -0.39134700 & -1.09851200 & $\mathrm{~N}$ & -2.61156700 & -0.68714100 & -0.28888600 \\
\hline $\mathrm{H}$ & 0.87884700 & 0.54863500 & -0.93378000 & $\mathrm{H}$ & -3.38118800 & -0.65533500 & -0.96558600 \\
\hline \multicolumn{4}{|c|}{$\mathrm{NH}_{2} \mathrm{NO}+\mathrm{HNO}_{3}$ (Figure 4) } & $\mathrm{O}$ & -1.09125600 & 0.49027300 & 0.64822600 \\
\hline \multicolumn{4}{|c|}{$\mathrm{NH}_{2} \mathrm{NO} \cdots \mathrm{HNO}_{3}, 4$} & $\mathrm{H}$ & -0.17294200 & 1.04655900 & 0.21614500 \\
\hline $\mathrm{N}$ & -2.49170100 & -0.24126700 & 0.00003300 & $\mathrm{O}$ & 1.04128700 & 1.11146600 & -0.15649900 \\
\hline $\mathrm{N}$ & -2.08254800 & 0.98545300 & -0.00023900 & $\mathrm{O}$ & 0.74070000 & -0.99000700 & 0.40464300 \\
\hline $\mathrm{O}$ & -1.60652600 & -1.08337500 & 0.00020500 & $\mathrm{H}$ & -0.39517100 & -0.43238700 & 0.62364600 \\
\hline $\mathrm{H}$ & -1.08298400 & 1.19384900 & -0.00025600 & $\mathrm{~N}$ & 1.51693400 & -0.07551900 & -0.04518000 \\
\hline $\mathrm{H}$ & -2.80106100 & 1.68856000 & -0.00038200 & $\mathrm{O}$ & 2.63596700 & -0.31481700 & -0.34798600 \\
\hline $\mathrm{O}$ & 1.03614500 & -1.10538900 & -0.00017700 & \multicolumn{4}{|c|}{$\mathrm{HNNOH}^{\cdots} \mathrm{HNO}_{3}, 12$} \\
\hline $\mathrm{H}$ & 0.04329900 & -0.96708800 & -0.00004800 & $\mathrm{~N}$ & -2.67378200 & 0.44670000 & -0.09744100 \\
\hline $\mathrm{O}$ & 0.85808700 & 1.07382900 & 0.00034000 & $\mathrm{~N}$ & -3.12595500 & -0.67503500 & -0.01358600 \\
\hline $\mathrm{N}$ & 1.60446100 & 0.11073800 & 0.00002400 & $\mathrm{H}$ & -4.12831700 & -0.57982400 & -0.19731800 \\
\hline $\mathrm{O}$ & 2.79095100 & 0.12746200 & -0.00012300 & $\mathrm{O}$ & -1.31464600 & 0.41140000 & 0.14322600 \\
\hline \multicolumn{4}{|c|}{$\mathrm{TS} 1 \cdots \mathrm{HNO}_{3}, 5$} & $\mathrm{H}$ & -0.99562600 & 1.31614600 & 0.03063900 \\
\hline $\mathrm{N}$ & 2.32762700 & -0.03334000 & 0.00596000 & $\mathrm{O}$ & 1.34646600 & 1.10163600 & -0.00722100 \\
\hline $\mathrm{N}$ & 1.64366000 & 1.00609400 & 0.13543200 & $\mathrm{O}$ & 1.02465700 & -1.06374400 & 0.04683600 \\
\hline $\mathrm{O}$ & 1.66495500 & -1.10793900 & -0.16583000 & $\mathrm{H}$ & 0.11897200 & -0.68326900 & 0.08387600 \\
\hline $\mathrm{H}$ & 0.50000100 & 1.03794100 & 0.02718700 & $\mathrm{~N}$ & 1.86043900 & 0.00646600 & -0.01272400 \\
\hline $\mathrm{H}$ & 2.22009400 & 1.82760800 & 0.26980500 & $\mathrm{O}$ & 3.01603000 & -0.26178800 & -0.06420800 \\
\hline $\mathrm{O}$ & -0.76264400 & -1.04045600 & 0.17673500 & \multicolumn{4}{|c|}{$\mathrm{HNNOH}^{\cdots} \mathrm{HNO}_{3}, 19$} \\
\hline $\mathrm{H}$ & 0.58613300 & -0.99651400 & -0.05328400 & $\mathrm{~N}$ & 2.58490700 & 0.53074600 & -0.00034100 \\
\hline $\mathrm{O}$ & -0.85622100 & 1.10799900 & -0.17190200 & $\mathrm{~N}$ & 1.37170500 & 0.62281200 & 0.00075300 \\
\hline $\mathrm{N}$ & -1.46385400 & 0.00380700 & 0.00211000 & $\mathrm{O}$ & 2.94919300 & -0.77872200 & -0.00029200 \\
\hline $\mathrm{O}$ & -2.65337500 & -0.04772500 & 0.00496900 & $\mathrm{H}$ & -0.04463500 & -0.46700500 & 0.00094900 \\
\hline \multicolumn{4}{|c|}{$\mathrm{HNNOH} \cdots \mathrm{HNO}_{3}, 6$} & $\mathrm{H}$ & 1.13306200 & 1.61634100 & 0.00065900 \\
\hline $\mathrm{N}$ & 2.51830500 & 0.11384300 & 0.00001000 & $\mathrm{O}$ & -1.50449000 & 1.13441100 & -0.00020200 \\
\hline $\mathrm{N}$ & 1.67380000 & 1.00720800 & -0.00005900 & $\mathrm{H}$ & 3.91297900 & -0.75595300 & -0.00154500 \\
\hline $\mathrm{O}$ & 2.01152500 & -1.11238300 & 0.00010900 & $\mathrm{O}$ & -0.90554900 & -0.96749200 & 0.00073700 \\
\hline $\mathrm{H}$ & -0.05684700 & 1.01222500 & -0.00001300 & $\mathrm{~N}$ & -1.87218500 & -0.01789100 & -0.00006600 \\
\hline $\mathrm{H}$ & 2.18149700 & 1.89212600 & -0.00012800 & $\mathrm{O}$ & -2.98820400 & -0.43107800 & -0.00055300 \\
\hline $\mathrm{O}$ & -0.83267500 & -1.03381200 & -0.00017700 & \multicolumn{4}{|c|}{$\mathrm{TS} 3 \cdots \mathrm{HNO}_{3}, 20$} \\
\hline $\mathrm{H}$ & 1.02932200 & -1.06277900 & 0.00009400 & $\mathrm{~N}$ & -2.29665600 & 0.56524700 & 0.00015200 \\
\hline $\mathrm{O}$ & -1.05486300 & 1.14090300 & 0.00003900 & $\mathrm{~N}$ & -1.10808000 & 0.32584800 & -0.00046000 \\
\hline $\mathrm{N}$ & -1.60090500 & -0.08787200 & -0.00001800 & $\mathrm{O}$ & -3.01939000 & -0.56412600 & 0.00041800 \\
\hline $\mathrm{O}$ & -2.78553300 & -0.12893500 & 0.00009400 & $\mathrm{H}$ & -0.53826700 & -0.61000500 & -0.00066000 \\
\hline \multicolumn{4}{|c|}{$\mathrm{HNNOH} \cdots \mathrm{HNO}_{3}, 10$} & $\mathrm{H}$ & -0.29046400 & 1.05086600 & -0.00053400 \\
\hline $\mathrm{N}$ & 2.63131700 & 0.49943100 & 0.00542300 & $\mathrm{O}$ & 1.23234300 & 1.10821800 & -0.00019300 \\
\hline $\mathrm{N}$ & 3.34810100 & -0.47691100 & -0.09789300 & $\mathrm{H}$ & -3.93932900 & -0.26854300 & 0.00074300 \\
\hline $\mathrm{H}$ & 4.30367000 & -0.12262700 & -0.18637700 & $\mathrm{O}$ & 0.93680800 & -1.06227100 & -0.00068700 \\
\hline $\mathrm{O}$ & 1.31776900 & 0.14513500 & 0.13322800 & $\mathrm{~N}$ & 1.72702200 & -0.06523700 & -0.00001100 \\
\hline $\mathrm{H}$ & -0.31805100 & 0.89228100 & 0.03216500 & $\mathrm{O}$ & 2.91424600 & -0.22598600 & 0.00079600 \\
\hline $\mathrm{O}$ & -1.27699100 & 1.09815800 & -0.01488200 & \multicolumn{4}{|c|}{$\mathrm{HNNOH}^{\cdots} \mathrm{HNO}_{3}, 21$} \\
\hline $\mathrm{O}$ & -1.18407000 & -1.08736200 & 0.04421700 & $\mathrm{~N}$ & 2.44775800 & -0.55055700 & 0.00011300 \\
\hline $\mathrm{H}$ & 1.24812900 & -0.82804300 & 0.09847600 & $\mathrm{~N}$ & 1.34250700 & -0.05127100 & -0.00021600 \\
\hline $\mathrm{N}$ & -1.89724500 & -0.11325600 & -0.01153600 & $\mathrm{O}$ & 3.44837800 & 0.39057800 & 0.00006800 \\
\hline $\mathrm{O}$ & -3.08282700 & -0.06923800 & -0.06459000 & $\mathrm{H}$ & 1.36476400 & 0.98102300 & -0.00044700 \\
\hline \multicolumn{4}{|c|}{$\mathrm{TS}_{2} \cdots \mathrm{HNO}_{3}, 11$} & $\mathrm{H}$ & -0.28289900 & -0.75249100 & -0.00014100 \\
\hline $\mathrm{N}$ & -2.14312300 & 0.42921000 & -0.27468800 & $\mathrm{O}$ & -1.23481900 & -1.04668800 & -0.00008100 \\
\hline
\end{tabular}




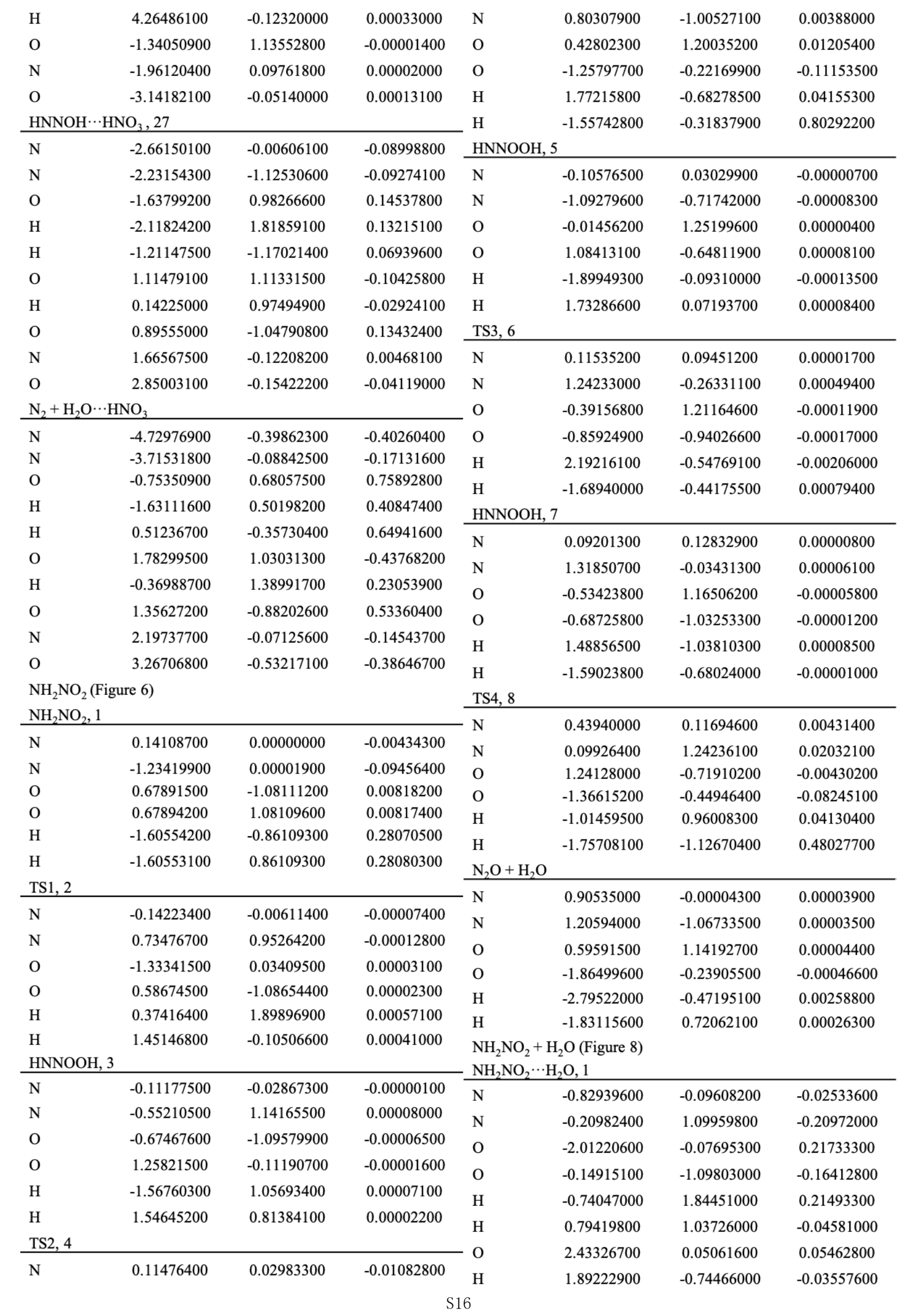




\begin{tabular}{|c|c|c|c|c|c|c|c|}
\hline \multirow{2}{*}{\multicolumn{4}{|c|}{$\begin{array}{l}\mathrm{H} \\
\mathrm{TS} 1 \cdots \mathrm{H}_{2} \mathrm{O}, 2\end{array}$}} & \multirow{3}{*}{\multicolumn{2}{|c|}{$\begin{array}{lr}\mathrm{H} & 0.84015200 \\
\mathrm{H} & 2.98997200 \\
\mathrm{HNNOOH} \cdots & \mathrm{H}_{2} \mathrm{O}, 25 \\
\end{array}$}} & \multirow{3}{*}{$\begin{array}{l}0.81059200 \\
0.09907800\end{array}$} & \multirow{3}{*}{$\begin{array}{l}-0.01642900 \\
0.62748900\end{array}$} \\
\hline & & & & & & & \\
\hline $\mathrm{N}$ & -0.72425500 & -0.01996900 & 0.00210000 & & & & \\
\hline $\mathrm{N}$ & -0.05889500 & 1.09407100 & -0.00708400 & $\mathrm{~N}$ & -0.87936500 & -0.15933900 & 0.00127500 \\
\hline $\mathrm{O}$ & -1.92922300 & -0.08299500 & 0.02195200 & $\mathrm{~N}$ & -0.22980600 & -1.20289500 & -0.01052400 \\
\hline $\mathrm{O}$ & -0.02216000 & -1.10404400 & -0.01109700 & $\mathrm{O}$ & -2.08210400 & 0.00393900 & 0.02651500 \\
\hline $\mathrm{H}$ & -0.71077200 & 1.86687800 & -0.00649000 & $\mathrm{O}$ & -0.14177800 & 1.04910000 & -0.01589800 \\
\hline $\mathrm{H}$ & 1.26580900 & 0.79999700 & -0.02818700 & $\mathrm{H}$ & 0.76602400 & -0.96300100 & -0.03304900 \\
\hline $\mathrm{O}$ & 2.08892600 & 0.00765800 & -0.08693100 & $\mathrm{H}$ & -0.84836800 & 1.71186400 & -0.01817300 \\
\hline $\mathrm{H}$ & 1.20967300 & -0.72737000 & -0.03807700 & $\mathrm{O}$ & 2.52251500 & -0.04677000 & -0.06453300 \\
\hline $\mathrm{H}$ & 2.61699000 & -0.02317400 & 0.71625300 & $\mathrm{H}$ & 2.14075900 & 0.82461700 & 0.07527000 \\
\hline \multicolumn{4}{|c|}{$\mathrm{HNNOOH} \cdots \mathrm{H}_{2} \mathrm{O}, 3$} & $\mathrm{H}$ & 3.31672100 & -0.08798600 & 0.47202700 \\
\hline $\mathrm{N}$ & -0.82557600 & 0.03823900 & 0.00262100 & \multicolumn{4}{|c|}{$\mathrm{TS} 4 \cdots \mathrm{H}_{2} \mathrm{O}, 26$} \\
\hline $\mathrm{N}$ & -0.18950200 & 1.12687700 & -0.00368700 & $\mathrm{~N}$ & -0.84345700 & -0.30344700 & -0.00648900 \\
\hline $\mathrm{O}$ & -2.01955200 & -0.14646300 & 0.01654900 & $\mathrm{~N}$ & -0.11835300 & -1.21671700 & -0.06315300 \\
\hline $\mathrm{O}$ & -0.07494500 & -1.08428300 & -0.00692200 & $\mathrm{O}$ & -1.96666300 & 0.04637800 & 0.07967300 \\
\hline $\mathrm{H}$ & -0.90086900 & 1.85484200 & 0.00123100 & $\mathrm{O}$ & 0.12642100 & 1.22927300 & -0.12774900 \\
\hline $\mathrm{H}$ & 1.90613800 & 0.82837500 & -0.00941600 & $\mathrm{H}$ & 1.04070500 & -0.87738400 & -0.05894400 \\
\hline $\mathrm{O}$ & 2.37161000 & -0.01782800 & -0.08397900 & $\mathrm{H}$ & -0.36794000 & 1.86599300 & 0.39590400 \\
\hline $\mathrm{H}$ & 0.87599000 & -0.79159500 & -0.02344900 & $\mathrm{O}$ & 2.09239200 & -0.12976000 & -0.03346700 \\
\hline $\mathrm{H}$ & 3.00737800 & -0.05884300 & 0.63390900 & $\mathrm{H}$ & 1.44638000 & 0.69719400 & 0.02190900 \\
\hline \multicolumn{4}{|c|}{$\mathrm{HNNOOH}^{\cdots} \mathrm{H}_{2} \mathrm{O}, 16$} & $\mathrm{H}$ & 2.59632200 & -0.21178400 & 0.78096100 \\
\hline $\mathrm{N}$ & 0.81287000 & -0.03376800 & 0.00204400 & \multicolumn{4}{|c|}{$\mathrm{N}_{2} \mathrm{O}+\mathrm{H}_{2} \mathrm{O} \cdots \mathrm{H}_{2} \mathrm{O}$} \\
\hline $\mathrm{N}$ & 2.03695700 & 0.15566800 & 0.01798900 & $\mathrm{~N}$ & 1.34369800 & -0.49910500 & 0.00591500 \\
\hline $\mathrm{O}$ & 0.18924400 & -1.10427100 & -0.00559600 & $\mathrm{~N}$ & 0.50240600 & -1.22356200 & 0.05873300 \\
\hline $\mathrm{O}$ & 0.08011300 & 1.09863500 & -0.00747700 & $\mathrm{O}$ & 2.24474500 & 0.24897100 & -0.05070500 \\
\hline $\mathrm{H}$ & 2.44894100 & -0.77730900 & 0.02256400 & $\mathrm{O}$ & -0.51602000 & 1.53851800 & 0.09043400 \\
\hline $\mathrm{H}$ & -0.86634400 & 0.79436200 & -0.02181600 & $\mathrm{H}$ & -1.74833000 & -1.10018900 & -0.08210200 \\
\hline $\mathrm{O}$ & -2.35097500 & -0.00032000 & -0.08128000 & $\mathrm{H}$ & -0.64623700 & 2.42180500 & -0.25720400 \\
\hline $\mathrm{H}$ & -1.88719000 & -0.84682300 & -0.01953200 & $\mathrm{O}$ & -2.49027000 & -0.48773500 & -0.12625200 \\
\hline $\mathrm{H}$ & -2.99124300 & 0.02412200 & 0.63337300 & $\mathrm{H}$ & -1.35859000 & 1.07405700 & -0.01264200 \\
\hline \multicolumn{4}{|c|}{$\mathrm{TS} 3 \cdots \mathrm{H}_{2} \mathrm{O}, 17$} & $\mathrm{H}$ & -3.07720400 & -0.73503900 & 0.59158700 \\
\hline $\mathrm{N}$ & -0.71203400 & -0.04229300 & 0.00129300 & \multicolumn{4}{|c|}{$\mathrm{NH}_{2} \mathrm{NO}_{2}+\mathrm{HCOOH}$ (Figure 8) } \\
\hline $\mathrm{N}$ & -1.94516800 & -0.10493900 & 0.02322800 & \multicolumn{4}{|c|}{$\mathrm{NH}_{2} \mathrm{NO}_{2} \cdots \mathrm{HCOOH}, 7$} \\
\hline $\mathrm{O}$ & -0.06546500 & 1.09296100 & -0.01133200 & $\mathrm{~N}$ & -1.57206500 & -0.10437900 & 0.01141600 \\
\hline $\mathrm{O}$ & -0.01088100 & -1.12227200 & -0.00962900 & $\mathrm{~N}$ & -1.06742700 & 1.11373700 & 0.28407300 \\
\hline $\mathrm{H}$ & -2.26858400 & 0.86263300 & 0.02560300 & $\mathrm{O}$ & -0.79212300 & -1.05201000 & 0.04264500 \\
\hline $\mathrm{H}$ & 1.23092600 & -0.72933200 & -0.03496500 & $\mathrm{O}$ & -2.75421200 & -0.19601200 & -0.20167400 \\
\hline $\mathrm{O}$ & 2.05969100 & 0.03936400 & -0.08549100 & $\mathrm{H}$ & -0.05832000 & 1.18285600 & 0.11661900 \\
\hline $\mathrm{H}$ & 1.19111000 & 0.76786100 & -0.03393300 & $\mathrm{H}$ & -1.69355400 & 1.84527200 & -0.01456000 \\
\hline $\mathrm{H}$ & 2.58020900 & 0.04903700 & 0.72326000 & $\mathrm{C}$ & 2.41652500 & 0.12056400 & -0.06525900 \\
\hline \multicolumn{4}{|c|}{$\mathrm{HNNOOH} \cdots \mathrm{H}_{2} \mathrm{O}, 18$} & $\mathrm{H}$ & 3.50917000 & 0.14425000 & -0.10870800 \\
\hline $\mathrm{N}$ & -0.81575700 & -0.11150000 & 0.00295000 & $\mathrm{O}$ & 1.96430400 & -1.10139200 & 0.08633100 \\
\hline $\mathrm{N}$ & -2.05177800 & -0.03876700 & 0.01471200 & $\mathrm{H}$ & 0.98010100 & -1.09307900 & 0.10701200 \\
\hline $\mathrm{O}$ & -0.11989700 & 1.07223800 & -0.00567500 & $\mathrm{O}$ & 1.73701800 & 1.11589100 & -0.14945600 \\
\hline $\mathrm{O}$ & -0.14830200 & -1.13829100 & -0.00116600 & \multicolumn{4}{|c|}{$\mathrm{TS} 1 \cdots \mathrm{HCOOH}, 8$} \\
\hline $\mathrm{H}$ & -2.28531400 & 0.95260600 & 0.01305600 & $\mathrm{~N}$ & -1.44327800 & -0.00311900 & -0.00001900 \\
\hline $\mathrm{H}$ & 1.91287600 & -0.79934200 & -0.03227200 & $\mathrm{~N}$ & -0.73969500 & 1.07931700 & -0.00016500 \\
\hline $\mathrm{O}$ & 2.34508100 & 0.06467100 & -0.08259200 & $\mathrm{O}$ & -0.81122700 & -1.12670700 & -0.00017200 \\
\hline
\end{tabular}




\begin{tabular}{|c|c|c|c|c|c|c|c|}
\hline $\mathrm{O}$ & -2.64746500 & -0.01015200 & 0.00021000 & $\mathrm{H}$ & 0.04258800 & 0.55456300 & 0.45380200 \\
\hline $\mathrm{H}$ & 0.55617900 & 1.09723800 & -0.00006300 & $\mathrm{C}$ & 2.52063300 & 0.15375500 & -0.12087400 \\
\hline $\mathrm{H}$ & -1.36582100 & 1.87419200 & -0.00006800 & $\mathrm{H}$ & 3.58284100 & 0.28620700 & -0.34271200 \\
\hline $\mathrm{C}$ & 2.23533400 & -0.04173000 & 0.00005600 & $\mathrm{O}$ & 1.79447800 & 1.05517100 & 0.20875500 \\
\hline $\mathrm{H}$ & 3.32539300 & -0.11126500 & 0.00006300 & $\mathrm{O}$ & 2.16225400 & -1.11221300 & -0.26487700 \\
\hline $\mathrm{O}$ & 1.59168500 & -1.11419500 & 0.00005700 & $\mathrm{H}$ & 1.21479800 & -1.21576700 & -0.06602100 \\
\hline $\mathrm{H}$ & 0.37878900 & -1.04464000 & -0.00007400 & $\mathrm{O}$ & -1.87626400 & 1.16626200 & -0.36284600 \\
\hline $\mathrm{O}$ & 1.73879000 & 1.11373700 & 0.00004100 & \multicolumn{4}{|c|}{$\mathrm{HNNOOH} \cdots \mathrm{HCOOH}, 22$} \\
\hline \multicolumn{4}{|c|}{$\mathrm{HNNOOH} \cdots \mathrm{HCOOH}, 9$} & $\mathrm{~N}$ & 1.52827900 & -0.04566900 & 0.00002200 \\
\hline $\mathrm{N}$ & -1.54036800 & 0.05670800 & -0.00000700 & $\mathrm{~N}$ & 2.76350900 & -0.06397200 & -0.00017000 \\
\hline $\mathrm{N}$ & -0.75900500 & 1.05457400 & -0.00011900 & $\mathrm{O}$ & 0.98335100 & 1.17315200 & -0.00026900 \\
\hline $\mathrm{O}$ & -0.97195700 & -1.15158500 & -0.00008800 & $\mathrm{O}$ & 0.75141100 & -1.02431800 & 0.00047900 \\
\hline $\mathrm{O}$ & -2.74500300 & 0.06685900 & 0.00013300 & $\mathrm{H}$ & -0.01517800 & 1.05439500 & 0.00002700 \\
\hline $\mathrm{H}$ & 0.95565100 & 1.12827400 & -0.00003700 & $\mathrm{H}$ & 3.01786100 & -1.05184700 & 0.00008300 \\
\hline $\mathrm{H}$ & -1.35723900 & 1.87694300 & -0.00003200 & $\mathrm{C}$ & -2.34372500 & 0.11902000 & -0.00000500 \\
\hline $\mathrm{C}$ & 2.35308100 & -0.13622300 & 0.00004800 & $\mathrm{H}$ & -3.43341300 & 0.20087300 & -0.00001700 \\
\hline $\mathrm{H}$ & 3.44147200 & -0.23565900 & 0.00011100 & $\mathrm{O}$ & -1.93620600 & -1.12085500 & -0.00037700 \\
\hline $\mathrm{O}$ & 1.62136700 & -1.10595200 & 0.00001000 & $\mathrm{H}$ & -0.94627100 & -1.13462000 & -0.00024100 \\
\hline $\mathrm{H}$ & 0.03205600 & -1.04335000 & -0.00005900 & $\mathrm{O}$ & -1.62395200 & 1.09509200 & 0.00031900 \\
\hline $\mathrm{O}$ & 1.95874100 & 1.10469600 & 0.00002200 & \multicolumn{4}{|c|}{ TS3 $\cdots \mathrm{HCOOH}, 23$} \\
\hline \multicolumn{4}{|c|}{$\mathrm{HNNOOH} \cdots \mathrm{HCOOH}, 13$} & $\mathrm{~N}$ & 1.41262100 & -0.04146100 & -0.00519600 \\
\hline $\mathrm{N}$ & 1.70799500 & -0.00605100 & 0.01411900 & $\mathrm{~N}$ & 2.64582300 & -0.08975100 & -0.01883900 \\
\hline $\mathrm{N}$ & 1.84609200 & 1.17658700 & -0.36084500 & $\mathrm{O}$ & 0.73441400 & -1.11216300 & -0.19818500 \\
\hline $\mathrm{H}$ & 2.78148200 & 1.22949200 & -0.76286300 & $\mathrm{O}$ & 0.76563200 & 1.06698600 & 0.20189800 \\
\hline $\mathrm{O}$ & 0.49549200 & -0.27571000 & 0.59211400 & $\mathrm{H}$ & -0.49323700 & -1.04876500 & 0.05369600 \\
\hline $\mathrm{H}$ & -1.20133100 & -1.20257800 & -0.09191200 & $\mathrm{H}$ & 2.96116500 & 0.86868200 & 0.13142200 \\
\hline $\mathrm{C}$ & -2.50416600 & 0.17060900 & -0.12876900 & $\mathrm{C}$ & -2.19735400 & 0.02327200 & 0.00010900 \\
\hline $\mathrm{H}$ & -3.56133200 & 0.31252900 & -0.36806400 & $\mathrm{H}$ & -3.28815300 & 0.03848700 & 0.00027800 \\
\hline $\mathrm{O}$ & -2.14444700 & -1.09030200 & -0.30675800 & $\mathrm{O}$ & -1.60572400 & 1.11167000 & -0.19958500 \\
\hline $\mathrm{O}$ & -1.78400400 & 1.05978800 & 0.24526500 & $\mathrm{H}$ & -0.45878400 & 1.04421300 & -0.05022600 \\
\hline $\mathrm{H}$ & -0.05158500 & 0.54111800 & 0.51262100 & $\mathrm{O}$ & -1.63756900 & -1.08196300 & 0.19992500 \\
\hline $\mathrm{O}$ & 2.45535300 & -0.95602200 & -0.04188200 & \multicolumn{4}{|c|}{ HNNOOH $\cdots \mathrm{HCOOH}, 24$} \\
\hline \multicolumn{4}{|c|}{ TS2 $\cdots \mathrm{HCOOH}, 14$} & $\mathrm{~N}$ & -1.52461300 & -0.12421000 & -0.00001000 \\
\hline $\mathrm{N}$ & 1.44154500 & -0.00835800 & 0.05213000 & $\mathrm{~N}$ & -2.75401100 & -0.24336600 & 0.00004600 \\
\hline $\mathrm{N}$ & 1.84339800 & 1.13926000 & -0.24529800 & $\mathrm{O}$ & -0.72304500 & -1.06384400 & -0.00009800 \\
\hline $\mathrm{H}$ & 2.69529300 & 0.98991800 & -0.78561900 & $\mathrm{O}$ & -1.00814900 & 1.12981900 & 0.00001800 \\
\hline $\mathrm{O}$ & 0.31580900 & -0.03488900 & 0.79174500 & $\mathrm{H}$ & 0.97832400 & -1.13496700 & 0.00002800 \\
\hline $\mathrm{H}$ & -0.62484000 & -0.90708900 & 0.31424300 & $\mathrm{H}$ & -3.13734200 & 0.70097000 & 0.00011500 \\
\hline $\mathrm{C}$ & -2.17715600 & 0.01333700 & -0.26778500 & $\mathrm{C}$ & 2.34006800 & 0.15911000 & 0.00001300 \\
\hline $\mathrm{H}$ & -3.18449000 & 0.03162100 & -0.67777300 & $\mathrm{H}$ & 3.42709400 & 0.27036700 & 0.00002700 \\
\hline $\mathrm{O}$ & -1.63051800 & -1.11024100 & -0.09387600 & $\mathrm{O}$ & 1.59517800 & 1.11695700 & -0.00004800 \\
\hline $\mathrm{O}$ & -1.63002100 & 1.11749000 & 0.00322700 & $\mathrm{H}$ & -0.00456600 & 1.04270100 & 0.00000200 \\
\hline $\mathrm{H}$ & -0.62732200 & 0.88003300 & 0.39372000 & $\mathrm{O}$ & 1.96682300 & -1.09052100 & 0.00006500 \\
\hline $\mathrm{O}$ & 1.92094200 & -1.09621200 & -0.23680600 & \multicolumn{4}{|c|}{$\mathrm{HNNOOH} \cdots \mathrm{HCOOH}, 28$} \\
\hline \multicolumn{4}{|c|}{ HNNOOH $\cdots \mathrm{HCOOH}, 15$} & $\mathrm{~N}$ & -1.68441500 & 0.14020200 & -0.01178400 \\
\hline $\mathrm{N}$ & -1.71545400 & 0.01491300 & 0.01167600 & $\mathrm{~N}$ & -1.18493900 & 1.25765200 & -0.07720700 \\
\hline $\mathrm{N}$ & -2.47455700 & -0.96675200 & -0.00934900 & $\mathrm{O}$ & -0.78781100 & -0.95104200 & 0.21702400 \\
\hline $\mathrm{H}$ & -3.34034700 & -0.62318700 & -0.42487200 & $\mathrm{O}$ & -2.83992700 & -0.20897600 & -0.10808300 \\
\hline $\mathrm{O}$ & -0.49216700 & -0.26690300 & 0.55506200 & $\mathrm{H}$ & -1.39622700 & -1.70493300 & 0.24910300 \\
\hline
\end{tabular}




\begin{tabular}{|c|c|c|c|c|c|c|c|}
\hline $\mathrm{H}$ & 1.11114600 & -1.10728300 & -0.09353200 & $\mathrm{H}$ & 1.75777700 & 1.85971400 & 0.00015900 \\
\hline $\mathrm{C}$ & 2.50164900 & 0.14894200 & -0.02373100 & $\mathrm{O}$ & -1.24030000 & -1.01590600 & 0.00010500 \\
\hline $\mathrm{H}$ & 3.59298700 & 0.20833800 & -0.06786600 & $\mathrm{H}$ & 0.47587300 & -1.06311100 & -0.00007700 \\
\hline $\mathrm{O}$ & 1.78581600 & 1.10247600 & 0.12420200 & $\mathrm{O}$ & -1.47983600 & 1.15762500 & -0.00012800 \\
\hline $\mathrm{H}$ & -0.16976700 & 1.18577300 & 0.05096500 & $\mathrm{~N}$ & -2.01467700 & -0.06748400 & 0.00001400 \\
\hline $\mathrm{O}$ & 2.08410200 & -1.10002300 & -0.15481100 & $\mathrm{O}$ & -3.19715400 & -0.12641600 & 0.00003900 \\
\hline \multicolumn{4}{|c|}{$\mathrm{N}_{2} \mathrm{O}+\mathrm{H}_{2} \mathrm{O} \cdots \mathrm{HCOOH}$} & \multicolumn{4}{|c|}{$\mathrm{HNNOOH} \cdots \mathrm{HNO}_{3}, 10$} \\
\hline $\mathrm{N}$ & 2.02444600 & -0.63213000 & 0.00050100 & $\mathrm{~N}$ & 2.28380600 & 0.02498600 & 0.00203700 \\
\hline $\mathrm{N}$ & 1.03650900 & -1.14028800 & 0.00304900 & $\mathrm{~N}$ & 2.81505800 & -1.07549300 & -0.24015100 \\
\hline $\mathrm{O}$ & 0.71682500 & 1.71590100 & -0.00040800 & $\mathrm{H}$ & 3.78763100 & -0.87330200 & -0.47029100 \\
\hline $\mathrm{O}$ & 3.07450600 & -0.11588800 & -0.00216900 & $\mathrm{O}$ & 0.94818900 & -0.06662800 & 0.33896100 \\
\hline $\mathrm{H}$ & 0.86982300 & 2.66176800 & -0.00209800 & $\mathrm{H}$ & -0.66257600 & 0.89476100 & 0.04050400 \\
\hline $\mathrm{H}$ & -0.24297200 & 1.59693800 & 0.00140700 & $\mathrm{O}$ & -1.60844500 & 1.11290100 & -0.07647400 \\
\hline $\mathrm{C}$ & -2.58778900 & -0.11223300 & -0.00194400 & $\mathrm{O}$ & -1.57633000 & -1.06817800 & 0.13779100 \\
\hline $\mathrm{H}$ & -3.67400200 & -0.24369100 & -0.00738700 & $\mathrm{H}$ & 0.70640300 & -1.00506900 & 0.25588600 \\
\hline $\mathrm{O}$ & -1.97326900 & -1.28320100 & -0.00130200 & $\mathrm{~N}$ & -2.25991100 & -0.08359100 & -0.02531100 \\
\hline $\mathrm{H}$ & -1.00831100 & -1.15450800 & 0.00373200 & $\mathrm{O}$ & -3.43679700 & -0.01585000 & -0.14883100 \\
\hline $\mathrm{O}$ & -2.04862200 & 0.96066500 & 0.00277400 & $\mathrm{O}$ & 2.71036700 & 1.15304200 & 0.00078700 \\
\hline \multicolumn{4}{|c|}{$\mathrm{NH}_{2} \mathrm{NO}_{2}+\mathrm{HNO}_{3}$ (Figure 8) } & \multicolumn{4}{|c|}{$\mathrm{TS} 2 \cdots \mathrm{HNO}_{3}, 11$} \\
\hline \multicolumn{4}{|c|}{$\mathrm{NH}_{2} \mathrm{NO}_{2} \cdots \mathrm{HNO}_{3}, 4$} & $\mathrm{~N}$ & 1.83459800 & -0.00321000 & -0.01069500 \\
\hline $\mathrm{N}$ & -2.01337300 & -0.09351700 & -0.00305800 & $\mathrm{~N}$ & 2.16114000 & 1.15584300 & -0.33183300 \\
\hline $\mathrm{N}$ & -1.54719600 & 1.11800000 & 0.37064600 & $\mathrm{H}$ & 2.95618300 & 1.04899000 & -0.96265400 \\
\hline $\mathrm{O}$ & -1.19177400 & -1.00209500 & -0.09215800 & $\mathrm{O}$ & 0.77957400 & -0.05322100 & 0.86424500 \\
\hline $\mathrm{O}$ & -3.19492400 & -0.21678600 & -0.17966100 & $\mathrm{H}$ & -0.12428500 & -0.84086500 & 0.45521400 \\
\hline $\mathrm{H}$ & -0.56085000 & 1.23682600 & 0.15325600 & $\mathrm{O}$ & -1.18908800 & -1.11174400 & 0.07761700 \\
\hline $\mathrm{H}$ & -2.20693600 & 1.84889600 & 0.15085500 & $\mathrm{O}$ & -1.19298600 & 1.08971900 & 0.21397000 \\
\hline $\mathrm{O}$ & 1.47582000 & -1.11401000 & 0.22564400 & $\mathrm{H}$ & -0.13041800 & 0.77837200 & 0.55720200 \\
\hline $\mathrm{H}$ & 0.49765600 & -0.98485500 & 0.12160300 & $\mathrm{~N}$ & -1.82055100 & 0.00147800 & -0.07381800 \\
\hline $\mathrm{O}$ & 1.29295100 & 1.00525000 & -0.28088700 & $\mathrm{O}$ & -2.92956400 & 0.02353500 & -0.45980400 \\
\hline $\mathrm{N}$ & 2.04416600 & 0.07728000 & -0.04034700 & $\mathrm{O}$ & 2.29109000 & -1.08144800 & -0.33794400 \\
\hline $\mathrm{O}$ & 3.22854600 & 0.10098900 & -0.01248800 & \multicolumn{4}{|c|}{$\mathrm{HNNOOH} \cdots \mathrm{HNO}_{3}, 12$} \\
\hline \multicolumn{4}{|c|}{$\mathrm{TS} \cdots \mathrm{HNO}_{3}, 5$} & $\mathrm{~N}$ & 2.29165300 & 0.00042100 & -0.00023000 \\
\hline $\mathrm{N}$ & -1.85185000 & -0.00507000 & -0.00393300 & $\mathrm{~N}$ & 2.70940700 & 1.16552900 & 0.01366200 \\
\hline $\mathrm{N}$ & -1.13924500 & 1.03564900 & 0.25754300 & $\mathrm{H}$ & 3.70567600 & 1.09908700 & -0.19594700 \\
\hline $\mathrm{O}$ & -1.20138600 & -1.08896800 & -0.32243600 & $\mathrm{O}$ & 0.94396800 & -0.07868900 & 0.27695900 \\
\hline $\mathrm{O}$ & -3.04558500 & -0.03437500 & 0.02494300 & $\mathrm{H}$ & 0.72369800 & -1.02134900 & 0.18875600 \\
\hline $\mathrm{H}$ & 0.04066900 & 1.04185000 & -0.02066300 & $\mathrm{O}$ & -1.58657900 & -1.06216400 & 0.10457300 \\
\hline $\mathrm{H}$ & -1.73254200 & 1.82160100 & 0.48972600 & $\mathrm{O}$ & -1.61307300 & 1.12364000 & -0.05104900 \\
\hline $\mathrm{O}$ & 1.15005000 & -1.00544500 & 0.33210200 & $\mathrm{H}$ & -0.66668900 & 0.89748100 & 0.04370800 \\
\hline $\mathrm{H}$ & -0.15121900 & -0.99138700 & -0.05521500 & $\mathrm{~N}$ & -2.26892800 & -0.07153100 & -0.02027700 \\
\hline $\mathrm{O}$ & 1.26248500 & 1.06452800 & -0.34167700 & $\mathrm{O}$ & -3.44758300 & 0.00428300 & -0.12123600 \\
\hline $\mathrm{N}$ & 1.86469900 & -0.01170700 & 0.00816800 & $\mathrm{O}$ & 2.84231600 & -1.06658800 & -0.20782200 \\
\hline $\mathrm{O}$ & 3.05041900 & -0.06126200 & 0.02628200 & \multicolumn{4}{|c|}{$\mathrm{HNNOOH} \cdots \mathrm{HNO}_{3}, 19$} \\
\hline \multicolumn{4}{|c|}{$\mathrm{HNNOOH} \cdots \mathrm{HNO}_{3}, 6$} & $\mathrm{~N}$ & -1.98477500 & -0.03187000 & 0.00235400 \\
\hline $\mathrm{N}$ & 2.00493300 & 0.04199100 & 0.00000300 & $\mathrm{~N}$ & -3.21529500 & -0.07468900 & -0.02268000 \\
\hline $\mathrm{N}$ & 1.19330600 & 1.01362200 & 0.00005300 & $\mathrm{O}$ & -1.46161900 & 1.20189300 & -0.05303200 \\
\hline $\mathrm{O}$ & 1.45703400 & -1.18419100 & -0.00014300 & $\mathrm{O}$ & -1.18006400 & -0.98544900 & 0.07340500 \\
\hline $\mathrm{O}$ & 3.20467100 & 0.07473800 & 0.00006300 & $\mathrm{H}$ & 0.45769600 & -1.02087500 & -0.01678600 \\
\hline $\mathrm{H}$ & -0.47390600 & 1.03969000 & -0.00005900 & $\mathrm{H}$ & -3.45605600 & -1.06546000 & 0.02239800 \\
\hline
\end{tabular}




\begin{tabular}{llllllll}
$\mathrm{O}$ & 1.24433700 & 1.01617100 & 0.06778400 & $\mathrm{H}$ & 0.10808300 & 2.16124100 & 0.67671200 \\
$\mathrm{H}$ & -0.48383900 & 1.07820200 & -0.00386000 & $\mathrm{O}$ & -0.69416400 & -0.06992300 & -1.02977800 \\
$\mathrm{O}$ & 1.44568700 & -1.15890700 & -0.05893700 & $\mathrm{~N}$ & -1.65003300 & -0.16578100 & -0.07066300 \\
$\mathrm{~N}$ & 2.00225400 & 0.05888800 & 0.00215600 & $\mathrm{O}$ & -2.39976200 & -1.07913900 & -0.19343500 \\
\cline { 5 - 8 } $\mathrm{O}$ & 3.18502200 & 0.09402100 & -0.01354000 & & & &
\end{tabular}

$\mathrm{TS} 3 \cdots \mathrm{HNO}_{3}, 20$

\begin{tabular}{lrrr}
\hline $\mathrm{N}$ & -1.80613500 & 0.03646500 & -0.00967500 \\
$\mathrm{~N}$ & -3.03038000 & 0.07823400 & -0.02904200 \\
$\mathrm{O}$ & -1.11940600 & 1.07119200 & -0.35715100 \\
$\mathrm{O}$ & -1.14155200 & -1.02892500 & 0.35679000 \\
$\mathrm{H}$ & -0.05905400 & -0.98356500 & -0.00251500 \\
$\mathrm{H}$ & -3.35443200 & -0.85117800 & 0.24374800 \\
$\mathrm{O}$ & 1.16989800 & 1.01882200 & 0.37959600 \\
$\mathrm{H}$ & -0.02970800 & 0.99739600 & 0.00625600 \\
$\mathrm{O}$ & 1.15182300 & -1.02621700 & -0.38019900 \\
$\mathrm{~N}$ & 1.82272900 & -0.01075600 & 0.00048400 \\
$\mathrm{O}$ & 3.00669800 & -0.02115400 & 0.00348200 \\
$\mathrm{HNNOOH} \cdots \mathrm{HNO}, 21$ & & \\
\hline $\mathrm{N}$ & -1.98416400 & -0.10345800 & 0.00014700 \\
$\mathrm{~N}$ & -3.20815700 & -0.23798400 & -0.00057800 \\
$\mathrm{O}$ & -1.16274100 & -1.02630700 & 0.00179900 \\
$\mathrm{O}$ & -1.47997500 & 1.16254400 & -0.00089100 \\
$\mathrm{H}$ & -0.49780500 & 1.05947500 & 0.00029900 \\
$\mathrm{H}$ & -3.61028500 & 0.69897300 & -0.00177200 \\
$\mathrm{O}$ & 1.47312600 & -1.15306200 & -0.00130100 \\
$\mathrm{H}$ & 0.48275500 & -1.03499500 & -0.00040500 \\
$\mathrm{O}$ & 1.22676100 & 1.02169300 & 0.00135500 \\
$\mathrm{~N}$ & 2.00397900 & 0.07697800 & -0.00001200 \\
$\mathrm{O}$ & 3.18579400 & 0.13610700 & -0.00033800 \\
$\mathrm{HNOOH}$
\end{tabular}

HNNOOH $\cdots \mathrm{HNO}_{3}, 27$

\begin{tabular}{lrrr}
\multicolumn{1}{l}{$\mathrm{HNNOOH} \cdots \mathrm{HNO}_{3}, 27$} \\
$\mathrm{~N}$ & 2.14270500 & 0.15439700 & -0.03018700 \\
$\mathrm{~N}$ & 1.67704400 & 1.28489900 & -0.14659600 \\
$\mathrm{O}$ & 3.27246700 & -0.24475200 & -0.17643400 \\
$\mathrm{O}$ & 1.22153600 & -0.87698100 & 0.34062400 \\
$\mathrm{H}$ & 1.79810700 & -1.65466900 & 0.40010100 \\
$\mathrm{H}$ & 0.67775800 & 1.25162300 & 0.06113600 \\
$\mathrm{O}$ & -1.54745600 & -1.13491900 & -0.21204000 \\
$\mathrm{H}$ & -0.59099600 & -0.99164000 & -0.05723600 \\
$\mathrm{O}$ & -1.36810200 & 0.99132200 & 0.26425600 \\
$\mathrm{~N}$ & -2.12123200 & 0.08223700 & -0.00318700 \\
$\mathrm{O}$ & -3.30025600 & 0.10832500 & -0.10943300 \\
$\mathrm{~N}_{2} \mathrm{O}+\mathrm{H}_{2} \mathrm{O} \cdots \mathrm{HNO}_{3}$ & & \\
\hline $\mathrm{N}$ & 1.89082100 & -0.73995100 & 0.26282200 \\
$\mathrm{~N}$ & 1.36357300 & -0.96770300 & 1.21360200 \\
$\mathrm{O}$ & 2.44448500 & -0.49431500 & -0.74911800 \\
$\mathrm{O}$ & 0.74985600 & 1.90552200 & 0.00432800 \\
$\mathrm{H}$ & 1.11705900 & 2.70718200 & -0.37519800 \\
$\mathrm{H}$ & -0.13934500 & 0.71396000 & -0.76897400 \\
$\mathrm{O}$ & -1.63995500 & 0.67931400 & 0.79639400
\end{tabular}

